\title{
Un amour de serpent
}

Analyse de contes à travers quatre ethnies indochinoises

\section{Jacques Dournes}

\section{(2) OpenEdition}

Journals

Édition électronique

URL : https://journals.openedition.org/clo/631

DOI : $10.4000 /$ clo.631

ISSN : 2266-1816

Éditeur

INALCO

\section{Édition imprimée}

Date de publication : 1 janvier 2009

Pagination : 65-105

ISBN : 978-2-85831-188-0

ISSN : 0396-891X

Référence électronique

Jacques Dournes, « Un amour de serpent », Cahiers de littérature orale [En ligne], 66 | 2009, mis en ligne le 25 février 2013, consulté le 30 juin 2021. URL : http://journals.openedition.org/clo/631 ; DOI :

https://doi.org/10.4000/clo.631

Ce document a été généré automatiquement le 30 juin 2021.

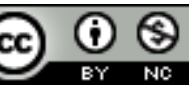

Cahiers de littérature orale est mis à disposition selon les termes de la Licence Creative Commons Attribution - Pas d'Utilisation Commerciale 4.0 International. 


\section{Un amour de serpent}

Analyse de contes à travers quatre ethnies indochinoises

\section{Jacques Dournes}

\section{NOTE DE L'ÉDITEUR}

Article paru pour la première fois dans Cahiers de littérature orale n² 2, 1977, p. 103-164.

1 Comment un long python peut-il épouser une belle fille des hommes et pourquoi cette question (c'est-à-dire : que signifie-t-elle ?), c'est ce que nous verrons respectivement dans les textes que je vais présenter et dans l'analyse que j'en esquisserai. À défaut de nous faire entendre de beaux récits, en langue jörai ou en traduction, ce serpent pourrait, en posant des problèmes de sens, nous conduire à des réflexions sur l'amour et la mort.

\section{Les ethnies et leur littérature}

2 Entre la côte maritime, à l'est, aujourd'hui vietnamienne après avoir été cham, et la vallée du Mékong, à l'ouest, pays des Lao et Khmer, s'étend une région de plateaux plus ou moins bosselés où demeurent toujours les plus anciennes populations autochtones de la péninsule indochinoise. Elles se répartissent en deux grandes familles linguistiques:

- les Austroasiatiques, apparentés aux Khmers, divisés en deux groupes : celui du nord, dont les Böhnar, celui du sud avec les Ködu et Sré ;

- les Austronésiens, apparentés aux Cham et aux Malais-Indonésiens, installés entre les deux groupes précédents et dont les plus importants sont les Jörai.

Malgré cette différence de langue, telle qu'il n'y a pas d'inter-compréhension entre les locuteurs des divers groupes, bien que de nombreux mots soient communs à toutes les ethnies - notamment notre " python » qui se dit partout klan ou tlan - se manifeste une profonde unité culturelle. Il faut aussi noter que ces populations, que l'on a qualifiées 
de "primitives » après les avoir traitées de "sauvages ", n'ont jamais été fermées sur elles-mêmes; des facteurs économiques et politiques ont mis en mouvement le statisme apparent de leur répartition et de leur cloisonnement, d'où un entrecroisement d'influences (nord-sud entre Böhnar et Jörai, par exemple, est-ouest entre Cham et Khmer) et des emprunts qui ont contribué à tisser cette culture commune.

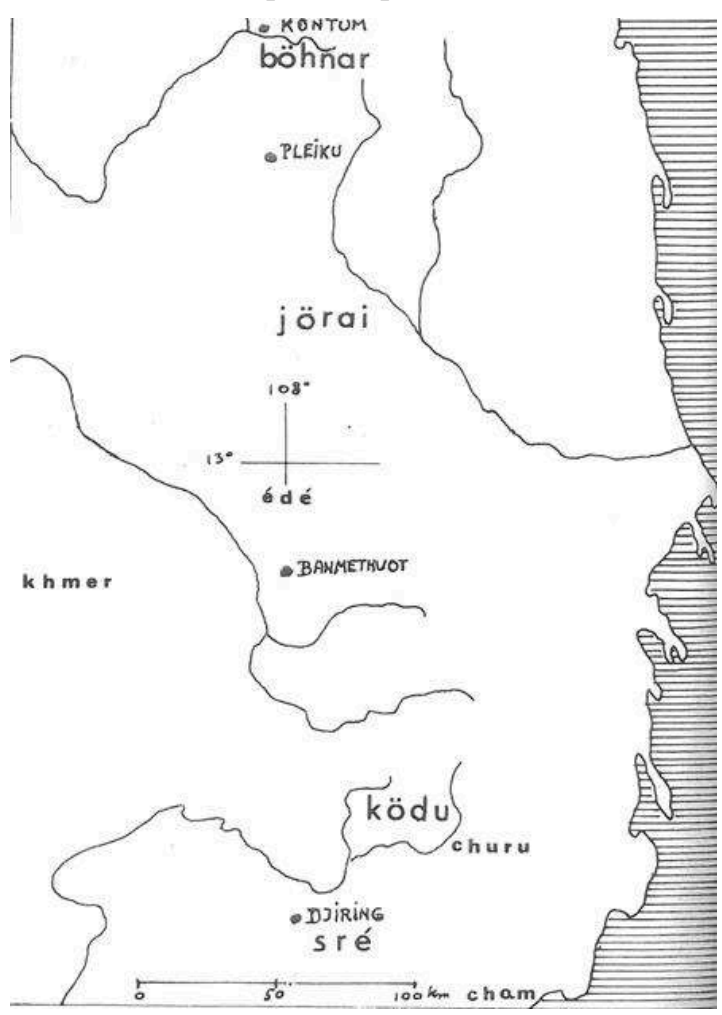

4 À côté de la vannerie et du tissage, et non sans lien avec eux, une des plus grandes richesses de ces populations - sans écriture - est leur littérature orale : mythes, contes et épopées, dits de justice et invocations rituelles, proverbes et chansons. La littérature orale des Böhnar (avec leurs sous-groupes röngao et jölöng, notamment) a été d'abord recueillie par E. Kemlin (1875-1925), puis abondamment par P. Guilleminet (entre 1932 et 1941), non encore publiée, elle m'a été communiquée, pour le sujet retenu ici, par l'obligeance de D. Léger ; celle des Ködu a été partiellement recueillie par A. Ferreiros (entre 1969 et 1971) ; celle des Sré et des Jörai par moi-même (respectivement de 1947 à 1954 et de 1955 à 1970), elle est en cours de publication (Bibliographie).

Une des caractéristiques de ces littératures, traditionnellement transmises de bouche à oreille et de génération en génération, est leur facture commune. Pour résumer en deux mots l'analyse qui a été faite (Dournes, 1976), je dirai que cette facture est une organisation de formules et d'énoncés. Que le conte soit récité ou chanté, on retrouve cette disposition interne. L'énoncé - au sens restrictif où je le définis ici, et qui n'est pas celui des linguistes - est une proposition exposant une situation, une action, racontées, lesquelles sont plus ou moins des clichés qu'on peut retrouver en plusieurs textes, quoi qu'il en soit de leurs transformations ; l'énoncé est la part la plus aisément traduisible et communicable; en fait, c'est ce qu'on trouve habituellement dans les traductions de mythes, surtout quand ils proviennent de cultures très éloignées de celle de la langue de traduction. La formule est une expression toute faite, récitée ; elle revient, à peine modifiée, en plusieurs textes; elle marque une articulation du récit, une ponctuation forte, permettant de découvrir le découpage intentionnel du texte et, par là, d'en 
comprendre le sens immédiat (pour les intéressés, conteur et auditeurs) - «immédiat ", car au delà de ce sens perçu, il y a le (ou les) sens caché(s) que peut révéler le système des variantes, et c'est ce que je chercherai dans l'analyse de celles-ci. Dans la formule, émise d'un seul souffle de voix, c'est la proposition elle-même qui est cliché; le plus souvent c'est une parole, qu'on pourrait appeler «performative » (Austin, 1970) : dans ce cas précis de style direct, par exemple l'Engagement imprudent (cf. infra, § 61) la formule déclenche la suite des actions, l'auditeur sait le résultat (rencontre + mariage), c'est « comme fait » quand on le dit.

6 C'est sur la base de cette facture en énoncés et en formules que le cadre de cet article ne me permet pas de justifier plus amplement (le sujet a déjà reçu un début de traitement dans Dournes, (1976, 304-305), notamment, et sera repris dans une publication ultérieure), que j'ai procédé à un premier découpage des textes, présentés ci-dessous en « séquences » numérotées (pour faciliter le rapprochement), évitant ainsi au maximum le risque d'interprétation prématurée et de subjectivité ethnocentrique que n'hésitent pas à prendre ceux qui aiment ou croient reconnaître les mêmes thèmes de par le monde, sans vérifier le sens original qu'ils peuvent avoir en telle ou telle culture.

\section{Les textes}

7 Les sigles $\mathrm{Mj}$ (suivis du numéro dans le corpus), Ms, Mk, Mb signifient: Mythe jörai, mythe sré, mythe ködu, mythe böhnar - les quatre ethnies dans lesquelles j'étudie des versions du « conte-type $»^{1}$ (choix gratuit et non jugement de valeur), telles que Mj 41a, pris comme mythe de référence, couvrant ainsi presque tous les plateaux sudindochinois. Bien entendu, il n'est pas question de traductions - certains textes sont très longs, et ce n'est pas le propos de CLO de les transcrire -, mais de schémas édifiés à partir de la structure du style oral de ces populations.

Mj 41a - (1) Mère Brousse cherche des ignames en forêt; Python, lové en haut d'un arbre, formule ceci : "Qu'il pleuve à verse afin que Mère Brousse monte s'abriter chez moi!»-(2) Après la nuit chez Python, Mère Brousse rentre chez elle, demande à sa petite-fille H'Bia si elle accepterait d'épouser Python ; H'Bia refuse, sa sœur cadette H'Lui $^{3}$ accepte - (3) Python et H'Lui ${ }^{2}$ sont mariés, ils vont se baigner, Python se défait de sa mue, la jette au fil de l'eau, H'Lui ${ }^{?}$ nage vers l'aval pour la rattraper, elle heurte un bois pointu, se tue ; Python la ressuscite - (4) Python, devenu jeune homme resplendissant, revient avec H'Lui ${ }^{2}-(5)^{2}-(6)$ L'aînée H'Kruah et la puinée H'Bia s'émerveillent, H'Bia désire un mari aussi beau que celui de la benjamine, elle veut épouser un Python qui lui apporte la richesse ; son beau-frère va lui en chercher un, après discussion il en décide un à venir chez H’Bia - (7) Ce serpent entre dans la maison des jeunes, avale tout ce qu'il trouve, monte chez H'Bia, avale tout y compris H'Bia, puis il disparaît - (8) Python (jeune homme) se met à sa recherche, le trouve, prétexte de lui chercher les poux pour l'assommer, lui ouvre le ventre, ressuscite H’Bia ; ils reviennent à la maison - (9) Par la suite, Python décide de descendre à la côte, chez les Yuan (Vietnamiens) - (10) Il formule cette recommandation à sa femme: "Quoi qu'on t'invite à aller voir d'extraordinaire, n'y va pas, ne sors pas!»-(11) H’Bia invite sa sœur à aller se balancer près de la rivière, H'Lui $^{\text {? }}$ cède, H'Bia la pousse à l'eau - (12) Dans l'eau, H'Lui $^{2}$ est avalée par un crocodile, elle reste vivante, elle engendre un garçon - (13) Le crocodile venant sur la berge, le garçon en fend la peau avec son petit couteau et en sort avec sa mère; de sa parole il crée une maison; son coq chante, le chant évoque un oranger et appelle le père absent - (14) Python, à la suite d'autres gens, 
revient de la côte - (15) Python et $\mathrm{H}^{\prime} \mathrm{Lui}^{3}$ se racontent leur histoire, ils se reconnaissent.

Dorénavant, pour simplifier, le personnage Python, qui se transforme en jeune homme, sera désigné $\mathrm{S}$, alors que $\mathrm{S}$ ' désignera le Python, vrai serpent, autre personnage - sans exclure (comme on le verra par la suite) l'ambiguïté tenant au fait que $S$ ' peut être vu comme l'aspect serpent de $\mathrm{S}$.

Mj 41b - (1) Mère-grand et son petit-fils Drit creusent le sol de la forêt pour chercher des ignames ; la petite $\mathrm{H}^{\prime} \mathrm{Lui}^{2}$ était restée à la maison ; Mère-grand appelle la pluie, elle se perd en brousse, tombe sur un jardin de bananiers, profère cette formule: «Si je vois le propriétaire, je lui fais des propositions pour ma petitefille ! » Du haut d'un arbre, $S$ a entendu, il descend, suit Mère-grand jusque chez elle - (2) H'Lui ${ }^{2}$ accepte d'épouser S - (3) H'Lui ${ }^{2}$ et S sont mariés, les richesses affluent dans leur maison - (4) S s'est défait de sa mue, c'est un beau jeune homme - (5) S va visiter ses parents; il revient, en beauté ; $\mathrm{H}^{\prime} \mathrm{Lui}^{?}$ ne le reconnaît pas; il revêt sa dépouille, ils sont heureux - (9) S doit descendre à la côte, chez les Cham - (10) S dit à $\mathrm{H}^{\prime} L u i^{2}$ : «Si on t'invite à sortir, n'y va pas, reste toujours à la maison!»-(11) $\mathrm{H}^{\prime}$ Bia complote la mort de $\mathrm{H}^{\prime} \mathrm{Lui}^{\mathrm{P}}$ pour épouser $\mathrm{S}, \mathrm{H}^{\prime} \mathrm{Lui}^{\mathrm{P}}$ sort avec elle, $\mathrm{H}^{\prime} \mathrm{Bia}$ la pousse à l'eau - (12) $\mathrm{H}^{\prime} L \mathrm{Li}^{2}$, enceinte et portant un œuf dans un pli de sa jupe, est avalée par un crocodile; là, elle enfante un garçon - (13) De son couteau le fils de $\mathrm{H}^{\prime} \mathrm{Lui}{ }^{\mathrm{P}}$ fend le ventre du crocodile, la mère et le fils sortent ; l'œuf devient coq qui chante - (14) S revient de chez les Cham, le chant du coq le guide - (15) S et H'Lui ${ }^{?}$ se reconnaissent ; $S$ tue H'Bia.

De ces deux variantes (différant surtout par l'absence de S' dans la seconde) du mythe de référence jörai, il convient de rapprocher le texte suivant :

Mj 115 - Enceinte, $\mathrm{H}^{\prime} \mathrm{Lui}^{\mathrm{P}}$ a envie d'oranges telles que celle qu'elle a vue emportée au fil de l'eau ; équipé comme un chasseur, son mari part à la recherche, en trouve, en cueille; enroulé en haut de l'arbre, $\mathrm{S}$ le traite de voleur, demande en mariage la fille à naître (1) - Cette fille, H'Bia, devenue grande, est contrainte d'épouser $\mathrm{S}$, après l'avoir refusé (2-3) - Elle voit $\mathrm{S}$ se débarrasser de sa mue, elle l'aime alors (4) $S$ veut visiter ses parents, $H^{\prime} B i a$ l'accompagne, $S$ rentre dans sa peau et fait mine d'enserrer H'Bia, effrayée elle rentre en courant, laissant tomber toutes ses parures pour aller plus vite; $\mathrm{S}$ les ramasse et les lui rapporte; rassurée, H'Bia repart avec lui ; chez $\mathrm{S}$, ce ne sont que serpents de toute espèce, c'est plein de richesses (5) H'Bia réside finalement chez son mari, elle est enceinte ; $\mathrm{S}$ descend chez les Yuan (9) - Pendant l'absence de S, des seigneurs locaux ravissent successivement H'Bia (cf. 11) - Enfin S revient ; victorieux des seigneurs, il reprend H'Bia (14-15).

10 Les noms des personnages féminins sont les mêmes qu'en $\mathrm{Mj} 41$, mais répartis autrement. H'Bia de $\mathrm{Mj} 115$ correspond à $\mathrm{H}^{\prime} \mathrm{Lui}^{2}$ de $\mathrm{Mj}$ 41, et aussi à sa sœur aînée (absente de Mj 115) puisqu'elle a commencé par jouer le rôle de l'aînée en refusant le mariage. La scène de la fuite de H'Bia (début de la séquence 5) se retrouve en Mj 44 : c'est alors Drit qui fuit devant un homme à l'aspect monstrueux; le monstre est en réalité un splendide adolescent qui fait le bonheur de ses amis. Mj 44 attire d'une part tous les mythes de métamorphoses (homme ou femme apparaissant d'abord sous forme animale ou monstrueuse, apportant amour et richesse à ceux qui les auront acceptés sous leur première forme) et, d'autre part, tout le cycle de Drit, le héros populaire dont l'existence est transformée par une fille-forêt, sortie d'un bambou ou d'une défense d'éléphant.

11 Ce rapide survol d'une partie de la mythologie jörai permet de pressentir une certaine équivalence entre Python, le monstre, la fée, tous issus du monde «sauvage » de la forêt. 
Bien qu'apparemment éloigné de cette série, où se découvre un système de transformations, un type de textes est à rapprocher :

Mj 77 - Le mari de H'Lui ${ }^{2}$ va descendre au pays des Yuan, il recommande à sa femme de ne pas sortir pendant son absence - Entraînée par des amies à aller cueillir des fleurs, $\mathrm{H}^{\prime} \mathrm{Lui}^{\mathrm{P}}$ est restée seule derrière; une fleur, trop haut placée, la tente beaucoup. $\mathrm{H}^{\prime} \mathrm{Lui}^{2}$ profère cette formule (cliché) : «Qu'une fille me la cueille, j'en ferai mon amie; qu'un garçon la cueille, j'en ferai mon époux!»-Singe se manifeste, cueille la fleur ; H'Lui ${ }^{2}$ se donne à lui ; tous les jours, elle revient à cet arbre pour le voir - Le beau-frère de $\mathrm{H}^{\prime} \mathrm{Lui}^{\mathrm{l}} \mathrm{s}^{\prime}$ 'aperçoit du manège, il se déguise, se fait passer pour $\mathrm{H}^{\prime} \mathrm{Lui}^{\mathrm{P}}$, attire Singe, prétexte de lui chercher les poux pour l'assommer - Sur ce, le mari revient, $\mathrm{H}^{\prime} \mathrm{Lui}^{?}$ accuse son beau-frère d'avoir abusé d'elle, celui-ci donne du singe à manger à son frère et dévoile tout - $\mathrm{H}^{\prime} \mathrm{Lui}{ }^{\mathrm{P}}$ s'enfuit en forêt...

Dans ce type, plus rare, l'animal est réel ; il s'agit ici d'un vrai singe, sans plus, analogue au personnage $S^{\prime}$. On a l'impression que ce mythe n'est pas raconté pour décrire une situation de bestialité, qui, sans être totalement inconnue, est jugée choquante, mais pour montrer que l'histoire tourne court et que l'héroïne disparait (il n'y a plus rien à dire sur elle) si l'ambiguïté du personnage " animal» est supprimée - ce qui revient aussi à mettre en valeur le jeu d'incertitudes déclenché par $\mathrm{S}$ (ou ses équivalents) qui éprouve son entourage par des apparences qu'il modifie à son gré, disposant librement de sa peau (enveloppe, mue). Le conte-type peut se passer de S', comme nous l'avons $\mathrm{vu}$; mais, s'il se passe de S, il est dénaturé - s'il ne tombe pas dans le non-sens. Je me garde bien de parler de "version canonique " et de "version déviante»; un tel jugement de valeur fausserait le système que forment ces mythes, système où chaque texte a sa place signifiante, où $\mathrm{Mj} 77$, par exemple, joue comme un vide dans une dentelle ou un silence dans une mélodie.

Passons maintenant à l'autre famille linguistique, celle des austro-asiatiques Sré, Ködu et Böhnar, du sud au nord.

Ms 74 - (1) Un homme va à la pêche avec ses deux filles, un serpent énorme menace d'avaler l'homme s'il ne lui donne pas une de ses deux filles - (2) L'aînée, Nga, s'enfuit ; la cadette, Ngi, veut sauver son père - (3) Ngi épouse $S$ - (4) S se dépouille de sa peau, se transforme en beau jeune homme, il apporte à sa femme des richesses merveilleuses - (6) Nga, jalouse de sa cadette, veut aussi un serpent; elle en trouve un en pêchant - (7) S' entraîne Nga sous l'eau - (8) Pour plaire à Ngi, S revêt sa mue qui fait de lui le maître des serpents, il va délivrer Nga - (5) S emmène Ngi et Nga chez ses parents, vers l'Océan - (11) Nga pousse Ngi à l'eau - (12) Ngi est avalée par une baleine - (13) S sauve Ngi - (11 bis) Nga pousse Ngi à l'eau - (12 bis) Ngi est prise dans une coquille marine - (13 bis) Des Cham ramassent la coquille et gardent Ngi chez eux - $(9,14-15) S$ retrouve Ngi et demeure avec elle chez les Cham.

Ms 75 - (1) Un chasseur va poser des collets ; un énorme serpent lui barre la route, réclame une jeune fille. L'homme propose ses sœurs : Ngi, Nga, Nai Tölui (qui a été mariée à un Cham); c'est la benjamine Nai Tölui ${ }^{i}$ que $\mathrm{S}$ veut, sans quoi il avalera tout le village - (2) Nai Tölui ${ }^{2}$ accepte pour sauver le village - (3) Comblée de présents et richement parée comme pour un rite funéraire, Nai Tölui ${ }^{7}$ est conduite à $S$ au pied de la montagne - (4) Nai Tölui ${ }^{2}$ est seule avec $S$, qui se débarrasse de sa mue - (5) Elle invite $S$ à venir visiter ses parents - (9) $S$ va descendre chez les Cham - (10) Il recommande au frère de veiller sur sa sœur : «Qu'elle ne sorte pas de la maison!» - (11) Nga, jalouse de sa sœur, l'entraine au bord de l'eau, l'égorge - (12) Dans l'eau, Nai Tölui ${ }^{2}$, qui garde avec elle une orange, un couteau et un œuf, est avalée par un crocodile ; là, elle accouche d'un garçon - (13) Le crocodile vient sur le sable; avec le couteau, le garçon fend la peau, sort avec sa mère; il plante l'orange qui devient un grand arbre, l'œuf est devenu un coq - (14) Nai Tölui ${ }^{2}$ est 
montée au ciel. S revient, rencontre le garçon - (15) Averti par son ancêtre Soleil, il reconnaît son fils; sur le chemin du retour (à la maison de la femme) $\mathrm{S}$ doit combattre le maître des serpents d'eau; à la maison, Nga essaie de se faire passer pour sa sœur; son neveu la bat. Nai Tölui ${ }^{2}$ redescend du ciel - (6) Nga veut un serpent pour elle, elle trouve un petit de python et l'élève; le serpent grossit de plus en plus - (7) S' avale Nga par les pieds, elle croit qu'il s'amuse ; mais il l'avale tout entière et l'emmène au fond de l'eau - (8) $S$ cherche Nga, il procède par divination avec riz saupoudré de curcuma rituel, il apprend où est Nga; il revêt son habit-serpent, trouve S' au fond de l'eau, le tente: "Lève la tête, je t'enfile un collier ! » S serre le coulant, attelle des éléphants qui tirent ainsi S' sur le rivage ; S lui fend la peau du ventre, en sort Nga, mangée par les vers. $S$ la découpe et la cuit, en fait une Nga toute neuve - (10') S interdit à Nga de se tenir au soleil - (11') Nga ne respecte pas l'interdit; au soleil elle fond; on la recouvre d'une hotte, elle devient termitière.

Je n'ai laissé de côté aucun détail; ceux que nous n'avons pas encore rencontrés se retrouveront plus loin. Les trois sœurs de Ms 75 semblent bien se réduire à deux : Nai Tölui $^{i}$ est la même que Ngi (dont on ne parle plus après ; mais c'est très important pour le nom du personnage (cf. infra). L'image du petit serpent qui grossit - mis dans une calebasse, il la brise ; mis dans une jarre, il la fait éclater - se retrouve textuellement dans un autre conte sré :

Ms 6 - Un homme pêche des anguilles de mer, il en attrape « une $»^{3}$ (appelons-le A). A lui dit de ne pas le manger. Il le met dans une calebasse, puis dans une jarre; A grossit de plus en plus, l'homme le met dans un étang. A lui dit: "De la tempête n'aie pas peur, du serpent de mer, n'aie pas peur!»A va combattre le serpent de mer; victorieux, il en revient avec des richesses. Le frère aîné de cet homme est jaloux, il en veut autant; A lui fait les mêmes recommandations, mais l'homme a peur ; A ne rapporte rien. L'aîné tue A, le cadet l'enterre. Là, pousse un bambou ; le cadet coupe ce bambou à vannerie, en fait une cage, celle-ci se remplit aussitôt de poules. L'aîné veut en faire autant : sa cage est pleine d'excréments.

Ce texte a un équivalent dans la littérature jörai :

Mj 27 - Mère Brousse va à la pêche, elle n'a qu'une anguille, elle la ramène à son petit-fils Drit. A grossit, ne tient plus dans les plus grandes marmites : on le met dans l'étang. A devient un éléphant. A dit à Drit de l'accompagner, il va combattre Dragon. « De la tempête, n'aie pas peur ; les poissons morts, ne les ramasse pas!»A va au fond de l'eau combattre Dragon, il en revient avec des richesses. Le seigneur du lieu veut posséder A ; A lui fait les mêmes recommandations ; mais le seigneur a peur; A est tué par Dragon. Drit plante en terre une défense de A, elle devient bambou géant dont les branches accrochent toutes les richesses du monde et les apportent à Drit. Le seigneur réclame ce bambou ; transplanté chez le seigneur, il ne lui rapporte qu'excréments... (L'histoire continue avec la cendre du bambou, etc.)

17 Ces deux versions «Anguille» nous préparent à analyser deux séries de correspondances $(\simeq)$ :

sœur aînée $\simeq$ frère aîné $\simeq$ seigneur (jaloux, puissants et lâches),

et surtout :

$\mathrm{A} \simeq \mathrm{S}$ (richesse) et $\mathrm{A} \simeq \mathrm{S}^{\prime}$ (désastre)

ce qui met sur la voie d'une signification de ce personnage central, ambigu.

18 On aura noté que les séquences $6,7,8$ ne viennent pas à la même place dans Ms 74 et Ms 75, S' intervenant avant ou après l'assassinat de l'héroïne par son aînée ; ce genre de déplacement d'un paquet de séquences est fréquent dans ces mythologies; dans le cas présent, la transformation n'a rien changé au sens (qui ne se lit pas dans la linéarité apparente). Les séquences $10^{\prime}$ et $11^{\prime}$ de Ms 75 reprennent, en les inversant, les 
séquences-types 10 et 11 : interdit et violation de l'interdit, suivie de conséquences opposées. La curieuse association femme + hotte + termitière (11') se retrouve dans un texte jörai et uniquement là, ce qui invite à y faire attention :

$\mathrm{Mj} 50$ - Frère et sœur font la rizière ensemble. Le frère mange des œufs de serpents ; tout son corps lui démange, il devient un énorme serpent, il cherche de l'eau où se cacher; ce n'est jamais assez profond, son dos émerge ; enfin, il plonge dans un trou d'eau et disparaît. Désolée, sa sœur s'assied sur sa hotte retournée, elle devient termitière. Chassant, le seigneur passe par là ; averti par la voix de la termitière, il libère la jeune fille et l'épouse. Elle réclame son frère et pleure au bord de l'eau; le serpent-frère montre sa tête, énorme comme un éléphant ; le seigneur et ses gens lui passent une grosse corde autour du cou et tirent, la corde casse ; c'est une tresse de trois cheveux de la sœur qui permet d'amener à terre le monstre. Le village est trop petit pour lui, on l'installe dans une vaste prairie qu'il recouvre entièrement ; alors, il redevient jeune homme ; débarrassé de sa mue, il vit auprès de sa sœur.

Je reviendrai sur la correspondance qui semble s'établir entre ce frère et notre $\mathrm{S}$ rapprochement que j'ai pu faire grâce à la termitière qui me faisait signe. Notons encore le lien logique et stable, présent dans la plupart des textes cités, et particulièrement net dans le mythe précédent, entre les aspects du personnage $S$ et le milieu naturel : dans les eaux, $\mathrm{S}$ est serpent (ou autre animal aquatique) et ne peut être que cela - au besoin le jeune homme revêt de dépouille de serpent pour aller combattre quelque maître du fond des eaux - ; sur terre ferme, le héros S est jeune homme, avec ou sans une apparence de serpent. Cette opposition sera à exploiter au niveau de l'analyse.

Ces excursus, peut-être déroutants au premier abord, servent à rappeler une vérité et son corollaire :

- dans la mythologie d'une culture (et celle-ci dépasse l'ethnie dans le cas présent) tout s'attire, tout se tient; quand on commence par un texte, on ne sait pas combien d'autres vont s'y raccrocher ;

- pour en faire une analyse solide, il conviendrait d'avoir en tête (pour y faire appel à toute occasion) toutes les mythologies d'une vaste région - ce qui ne laisse pas d'être très exigeant.

21 Écoutons maintenant les Ködu, proches des Sré par la langue et la localisation :

Mk 7 - (1) Un chasseur tire des oiseaux posés sur un banian; du haut de l'arbre un Python menace : l'homme doit lui donner une de ses filles, sinon il mourra - (2) Rentré chez lui, l'homme interroge ses deux filles : l'aînée, Nga, refuse, la cadette, Ngi, accepte. Le père conduit, richement parée, à $S$ - (4) Le banian rayonne d'une lumière merveilleuse ; le père et sa fille voient un splendide adolescent, jusqu'au buste, la moitié inférieure du corps est celle d'un python - (3) Le père parti, Ngi épouse S; c'est un vrai jeune homme ; l'arbre est devenu un palais - (5) Ngi a envie de revoir ses parents, $\mathrm{S}$ l'accompagne, en chemin il porte sa peau de serpent, il s'en dévêt à l'arrivée ; la nuit, dans la maison, brille une lumière étincelante, c'est celle qui émane du couple heureux - (6) Jalouse et dépitée, Nga veut aussi son python; elle en prend un petit, le met dans une calebasse ; il grossit, la calebasse craque, elle le met dans une jarre, la jarre éclate ; S' ne cesse de grossir - (7) S' tente d'avaler Nga, elle croit qu'il joue ; $S$ ' avale Nga tout entière et disparaît au fond des eaux - (8) $S$ part à la recherche de sa belle-sœur, il trouve S', le tire hors de l'eau, tente de lui ouvrir le ventre avec son couteau, il échoue, il y parvient avec une cypéracée coupante; il ressuscite Nga - (10') S interdit à Nga de sortir de la maison, sous aucun prétexte (v.g. voir des buffles s'entrecorner $=$ Mj 41b) $-\left(11^{\prime}\right)$ Nga sort voir des coqs se combattre ; sur le champ, elle est transformée en termitière ; ses parents la 
cherchent, ils entendent une voix venant de la termitière, puis plus rien, Nga est morte. De Ngi est issu le clan Kon Tac, qui a l'interdit clanique de manger des fruits de banian (figues).

\section{textes :}

Mk 9 -(1) Un couple a deux filles, la cadette s'appelle Pôt, elle est très belle. Un jeune homme, Bông, la désire; pour l'aborder (en l'éprouvant?) il se mue en serpent-dragon siffleur - (3) Pôt cède à ses instances; les parents leur font une maison à part - (4) $\mathrm{S}$ se dépouille de ses apparences, il est splendide; les parents de Pôt le surprennent, on fait la fête ; le riz pousse tout seul dans le champ - (6) La sœur aînée veut un serpent pour faire comme sa cadette, elle pêche un petit python, le met dans une calebasse, le nourrit - (7) S' grandit ; elle couche avec lui, S' l'enserre, se raidit, l'avale, l'emmène au fond des eaux - (8) $S$ va la chercher; par divination, avec riz saupoudré de curcuma, il apprend qu'elle est au fond d'une cuvette du fleuve; S plonge, rencontre S', lui passe le "collier de mariage", l'étrangle et le tire ainsi au sec, lui ouvre le ventre et en sort la fille, toujours vivante - $\left(10^{\prime}\right)$ S lui interdit de marcher sur les os de S' - (11') Sept jours après, la sœur de Pôt veut revoir le python qui l'a avalée, elle pose son pied sur ses os, elle meurt du coup.

Pour la première fois, nous voyons le héros $S$ se présenter d'emblée comme un jeune homme, il n'y a plus d'incertitude, l'auditeur sait tout de suite à quoi s'en tenir (mais dans les autres cas aussi, puisqu'il connaît le thème par cœur !). L'apparence du héros $S$ tient du serpent et du dragon aquatique, à connotation maléfique dans cette culture ce qui est aussitôt annulé par le fait que nous savons que c'est un beau jeune homme. La dernière séquence « attire » la finale d'un conte sré :

Ms 24 - En l'absence de son mari, Jung Wai, tentée par un oiseau, tombe à l'eau ; elle épouse le serpent-maître des eaux. Le mari revient, apprend d'un témoin ce qui s'est passé, il provoque le serpent, le tue. Par la suite, Jung Wai, partie pour la cueillette, se blesse au pied avec un os pointu du serpent mort ; elle en meurt.

Dans la culture commune de ces ethnies, une partie d'un animal dangereux (os, dent ou griffe) sert souvent d'instrument du destin; le fauve (ou le monstre) mort peut encore se venger efficacement - des récits de chasse le prouvent. En comparant les contextes, le serpent des eaux Ms 24 est équivalent du crocodile de Mj 41b, il n'est qu'animal, à la différence de S'; mais il s'en prend à une victime innocente - Jung Wai n'est pas Nga.

Je signale, en passant, que les récits ködu sont particulièrement riches en descriptions : la technique du python (Mk 9, séquence 7) qui s'enroule autour de sa proie, se raidit pour l'étouffer (il est constricteur comme le boa, serpent américain) et l'avaler à son aise, en commençant par les pieds; et, surtout, les effets de lumières produits par le couple d'amoureux, au point que les parents croient à un incendie. Jörai :

$\mathrm{Mb}$ (Guilleminet, s.d.) - (1) Un seigneur est parti se tailler une arbalète dans la forêt de montagne; ayant perdu son fer de hache, il entend un serpent siffler et formule alors ceci : "Que le siffleur me retrouve ma hache et je l'appellerai beau-frère ! ", puis : «Qu'il me la retrouve et je l'appellerai oncle !», enfin : « Je le marierai à ma fille!». Un jeune Python lui montre alors sa hache et réclame en mariage damoiselle Lui $^{2}$, la cadette. $\mathrm{S}$ suit le seigneur, ils montent ensemble à la maison commune (maison des jeunes) ; le seigneur ne veut pas rentrer chez lui pour dîner (2) Sa fille, dame K'Kruah, l'appelle; il lui propose d'épouser S, elle refuse. La seconde fille, damoiselle Püon, refuse de même. La benjamine, damoiselle Lui ${ }^{2}$, accepte, même si c'était une vipère, uniquement pour contenter son père. Le 
seigneur rentre enfin chez lui avec $\mathrm{S}$, lequel est mis à l'épreuve : on lui donne à choisir œufs cuits ou œufs crus, on lui présente une jarre de bière de riz avec chalumeau planté dedans; S mange et boit « comme nous autres Böhnar » - (3) Lui ${ }^{\text {? }}$ épouse $\mathrm{S}$; on leur fait une maison à part - $(4) \mathrm{S}$ et Lui ${ }^{?}$ vont au bain ; $\mathrm{S}$ fait mine de se baigner en aval, Lui ${ }^{2}$ lui dit de le faire en amont, comme un homme qu'il est. S se dépouille de sa mue, devient jeune homme resplendissant, une lumière éclatante émane de lui ; il jette sa peau au fil de l'eau, Lui ${ }^{2}$ la rattrape au passage, se tourne vers l'amont, ne reconnaît le jeune homme que lorsqu'il revêt à nouveau sa mue, puis $\mathrm{S}$ la jette définitivement. Retour à la maison du seigneur qui fête le couple merveilleux. Le seigneur leur donne des richesses à profusion - (6) Un autre seigneur, en aval du premier, entend parler de cela; il veut aussi un python à donner en mariage à sa fille; il part à la chasse avec ses chiens, trouve un gros python sauvage, le prend au cou par un nœud coulant, " pour te donner en mariage à ma fille "; S' se récuse : il ne sera jamais un homme. Le seigneur le traîne néanmoins jusque chez lui. La scène de la maison commune se reproduit; les filles refusent, seule la benjamine accepte. S' est mis à l'épreuve, il mange l'œuf cru, il boit la bière en engageant son cou dans l'ouverture de la jarre - (7) S' a faim, il commence à avaler la fille par les pieds, jusqu'à la taille; elle appelle son père qui lui dit que son mari ne fait que jouer. S' l'avale tout entière, puis disparaît dans l'océan, en aval (ou : vers l'ouest). Le seigneur cherche sa fille; dans la maison du jeune couple ce n'est qu'une grande flaque d'eau. S' reste introuvable. Le seigneur célèbre un rite funéraire pour sa fille disparue.

La différence la plus frappante de cette version consiste dans le rôle privilégié donné ici au père : c'est lui qui mène toute l'affaire; c'est lui, et non plus $S$, qui donne la richesse ; et surtout, à partir de la séquence 6, c'est lui qui se montre envieux (de son rival d'amont), amène $S$ ' chez lui et parle de " jeu » à sa fille terrifiée. Cela peut s'expliquer par le fait que la société böhnar est à filiation patrilinéaire et résidence patrilocale, alors que les autres sont matrilinéaires et matrilocales (ou uxorilocales). Ce n'est pas qu'un changement de ton, qui donnerait seulement au texte une coloration propre à l'ethnie böhnar; le sens même du conte se trouve modifié, l'accent étant mis sur rivalité des seigneurs et non plus sur l'ambiguïté du personnage S-S'.

Le lien déjà relevé entre $S$ ' et Eau se trouve ici fortement souligné : non seulement $S$ ' disparaît vers les bas-fonds océaniques, mais il laisse à sa place une flaque d'eau dans la maison, comme un signe de sa présence et de son milieu naturel. Notons encore l'insistance avec laquelle le texte böhnar oppose l'amont et l'aval, comme le plus au moins; l'homme doit se baigner en amont, la femme en aval - non que la femme serait « inférieure », mais parce qu'elle fait la lessive tout en se baignant, en aval d'elle l'eau est donc plus polluée (sans compter que la femme peut avoir ses règles, ce qui, chez toutes ces populations indochinoises, est plus sale qu' «impur»)-; le seigneur, qui réussit avec le gendre S, est situé en amont, son rival malchanceux est en aval ; enfin S' va se perdre dans la mer, en aval - le même mot désigne l'ouest, qui est aussi le domaine des trépassés, la mort par opposition à la vie du soleil levant et des sources des fleuves.

29 La première séquence, parole d'engagement imprudent à propos d'une hache, est une formule-cliché de la mythologie indochinoise ; elle introduit notamment un mythe jörai que nous sommes amené, par là, à rapprocher du conte-type « Python »:

Mj 30 - H'Bia est restée la dernière en forêt pour couper du bois de chauffage; sa hache se coince; elle s'engage imprudemment par cette formule : "Que quelqu'un m'aide! Si c'est une fille, j'en ferai mon amie ; si c'est un garçon, je l'épouserai !» Un homme se présente; il ramène H'Bia chez elle, l'épouse, puis l'invite chez lui. Il la fait attendre en forêt, prétextant de prévenir les siens. La Dame du Figuier chante 
comme un oiseau, elle veut prévenir H'Bia du danger. H’Bia maudit cet « oiseau ». Le mari (en fait, un éléphant) revient avec sa harde ; H’Bia est dévorée. Le mariéléphant va alors chercher la petite sœur de H'Bia, prétextant que celle-ci a eu un enfant et a besoin de l'aide de sa sœur; il emmène ainsi H'Lui ${ }^{2}$, la cadette, la fait attendre en forêt. La Dame du Figuier chante, $\mathrm{H}^{\prime}$ Lui $^{\mathrm{P}}$ la bénit, la Dame cache H'Lui ${ }^{2}$; les éléphants arrivent, ne trouvent pas la fille, se dévorent entre eux. La dame appelle des oiseaux qui vont ramener $\mathrm{H}^{\prime} \mathrm{Lui}^{\mathrm{P}}$ chez elle...

Il n'y a pas que les noms de H'Bia et de H'Lui? qui nous fassent signe; si l'éléphant se présente comme l'homologue de S', il semble bien qu'un rôle équivalent à celui de S soit joué par la mystérieuse Dame du Figuier, à la foi fée, arbre et oiseau (Dournes, 1975) ; cela doit aider à cerner le caractère du personnage $\mathrm{S}$ et à comprendre quelque chose à sa signification, par sa corrélation avec le monde de la forêt, des arbres et des hauteurs.

\section{Notes ethnolinguistiques}

Pour ne pas retarder la promenade, au tracé déjà bien sinueux, lacis de pistes à dégager, dans la brousse de ces textes, auxquels de nombreux autres étaient prêts à s'ajouter, je regroupe ici ce qui aurait pu être des notes de bas de page; un autre avantage de cette disposition est d'éviter les répétitions, chacune de ces notes regroupées s'appliquant à la presque totalité des textes, car il s'agit bien d'un ensemble réel, au sein d'une culture, et non d'une collection disparate de récits gratuitement rapprochés. Chaque note, à sa façon, soulignera cette communauté, tout en relevant des particularités ethniques.

\section{Le Python}

\begin{tabular}{|l|l|l|l|l|l|l|}
\hline & sré & ködu & jörai & böhnar & cham & kmer \\
\hline serpent (en général) & bəs & bəs & ala & bih & ala & pos $^{4}$ \\
\hline python & klan & klan & tlan & klan & klan & thlan \\
\hline
\end{tabular}

32 À la ligne "serpent ", les Austronésiens jörai et cham s'opposent nettement à tous les autres, qui sont austro-asiatiques (les finales $s$ et $h$ sont équivalentes); cependant, il faut noter que "venin de serpent" se dit en jörai : ia bih ( $i a=$ liquide). À la ligne "python ", il n'y a plus guère de différence, surtout quand on sait que le groupement de consonnes initiales $k l$ peut se réaliser $t l$ (« os », klay en édé, tlay en jörai ; le cas est très fréquent). Notre héros Python porte donc le même nom dans toute cette partie de la péninsule indochinoise (y compris le vietnamien : trăn).

bəs/ala sert de générique à tous les serpents, par exemple : bih tur (böhnar) = ala cətay (jörai) = Bungarus fasciatus (en sré, bas tur désigne le Naja; dans les dénominations zoologiques et botaniques, il est fréquent que le même mot, d'une langue à l'autre, s'applique à des espèces différentes). En sré et en jörai, praj est un classificateur des serpents venimeux, ainsi ala praJ dron (jörai) = Naja naja (en böhnar bih praJ ne s'applique qu'à une espèce de vipéridé ophiophage). En jörai seulement tlan, le python, n'est pas classé comme serpent, ala, il est à part ; en böhnar, le python se dit bih klan, en sré et en ködu, bəs klan ou klan tout court ; en jörai, s'il arrive de dire ala tlan, ala n'a pas 
cette fonction de classificateur, ala tlan désigne l'ensemble des reptiles (c'est-à-dire serpents + pythons) de même que ramah raman («rhinocéros + éléphant ») désigne les grands fauves. Dans les textes jörai, bien que le héros Python dise parfois, pour se rabaisser, qu'il n'est qu'un ala, il s'oppose à l'ensemble des ala de deux façons complémentaires : 1 il leur est supérieur, il leur commande, comme un maître des reptiles ; 2 il est situé en haut (montagne, arbre) alors que les ala ont une connotation aquatique (dans le mythe) - j'y reviendrai dans l'analyse.

Les Jörai distinguent quatre variétés de pythons, qu'ils nomment, par ordre de taille croissante : tlan pro', tlan kəniy, tlan bəya, tlan tuy - découpage qui ne recouvre pas tout à fait les espèces scientifiques: Python curtus, Python molurus, Python reticulatus. Le Python de nos contes est le tlan bəya ou le tlan tuy (c'est parfois précisé), c'est-à-dire le Python reticulatus, qui peut atteindre dix mètres de long, un des plus grands serpents du monde. Des chasseurs m'avaient apporté un bébé python, il faisait déjà quatre-vingtdix centimètres de long; le dessin (bəya) de ses écailles, taches brunes cernées de noir sur fond jaune, est d'une grande beauté. Ce type de python est capable d'avaler des proies énormes (par rapport à son diamètre), des cervidés, par exemple; quand il digère, allongé, il est assez facile de lui passer un coulant autour du cou et de le tirer derrière soi comme un jouet; son ventre gonflé lui ôte presque toute résistance. Quand il est à jeun, il est souvent lové (wEn) et fréquemment sur un arbre. Les Jörai n'en font rien ; ils semblent avoir récemment appris que la peau avait une valeur marchande.

Il importe maintenant de se demander ce que ces populations autochtones indochinoises pensent du serpent. C'est un animal comme les autres, il n'inspire pas de sentiments particuliers - rien notamment de cette horreur congénitale chez une bonne partie des judéo-chrétiens qui en ont fait l'Ennemi, un dieu en négatif, un sujet de frayeur, quand ce n'est pas un symbole phallique. Les Böhnar contractent des alliances rituelles avec des serpents, comme avec d'autres animaux (Kemlin, 1917, 102). En jörai, une série classificatoire associe python - serpent - termite - fourmi - scorpion scolopendre, qui ont en commun, sauf le python, leur morsure cuisante, parfois mortelle (Naja et Agkistrodon surtout) ; il faut faire attention où l'on met le pied. Mais ce n'est pas le cas de tous les serpents; il y a des couleuvres ratières que la population protège en raison de leur grande utilité dans les rizières en particulier - dans les bambous du toit de ma case j'en avais une, de deux mètres de long, familiarisée, sinon apprivoisée, qui m'a débarrassé des rats ; mes amis jörai la remarquaient à peine, mais les visiteurs étrangers étaient très mal à l'aise. Et il y a notre python, qui ne mord pas, il étouffe seulement sa proie avant de l'avaler; c'est le plus grand et un des plus beaux, la mythologie de toutes les ethnies le privilégie. Une fois seulement, dans une variante de Mj $4 l$ entendue de deux conteurs différents (donc assez stable), le héros $\mathrm{S}$ est un

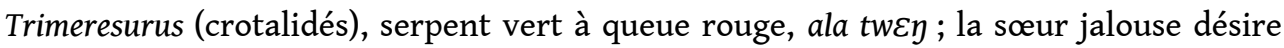
alors un Python, qui est ici S', parce que plus grand et censé apporter plus de bonheur. Cela nous avertit de ne pas donner trop d'importance à tel genre zoologique précis; ce qui est le plus pertinent, c'est la fonction de S et l'opposition S-S'.

La pensée mythique sré associe une vipère, bəs caylir, avec l'arc-en-ciel qui figurerait bəs caylir « s'accouplant avec le ciel » : bəs caplir tam tir mə tru:. Je ne puis m'étendre sur ce sujet qui nous emmènerait trop loin et nécessite un complément de recherche.

Un des phénomènes les plus remarquables - et pertinent pour notre analyse - observé chez le serpent, est sa mue. En böhnar, comme en jörai, tuh sor signifie «se dépouiller de sa mue», muer. En ces ethnies, une association d'images et d'idées se fait 
traditionnellement entre cette "mue " (et nous parlons bien de la mue de la voix) et une jeunesse toujours renouvelée, comme une victoire sur la mort ; j'ai même entendu dire que les serpents, s'ils n'étaient pas tués, ne mouraient pas. Le héros Python est toujours jeune, de même Drit, le petit pouilleux qui vit avec Mère Brousse. "Se débarrasser d'une apparence " pour vivre un amour hors du temps est une constante de ces mythologies et se dit encore tuh sor. La forme verbale tuh, qui en jörai et en böhnar désigne l'action de «mettre bas » (animaux), « se métamorphoser » (insectes), "muer» (serpents et arthropodes), a un sens particulier en böhnar quand elle se compose avec tədray, "s'équiper pour partir en guerre»; tuh tədray, "muer s'équiper ", se dit des guerriers qui, s'étant équipés et parés (comme le faisaient les Jörai) partaient en tête pour consulter les augures (Guilleminet, 1963). Manifeste est la correspondance entre la mue de notre héros, Python, qui, dépouillé de sa peau, est prêt à l'amour et celle des guerriers qui, rituellement parés, sont prêts au combat. Je ne vois pas qu'il y ait là quelque trace d' "initiation ", comme on en trouve ailleurs, mais bien plutôt le signe d'un changement d'état du jeune homme en quête du couple. Ce commentaire d'une expression böhnar, qui nous éclaire sur la mue de $\mathrm{S}$, montre, une fois de plus, qu'un mythe d'une ethnie ne s'explique bien que dans le contexte de la mythologie des ethnies voisines, du moment qu'elles font partie du même ensemble culturel. Ce qui est important, ce n'est pas que le héros $\mathrm{S}$ soit un python ou un autre serpent, c'est qu'il mue.

\section{Les autres caractères animaux}

Dans les textes présentés nous trouvons, toujours du côté des personnages masculins, outre Python (le héros S), d'une part le python S', d'autre part un serpent d'eau (Ms 75), une baleine (Ms 6), un dragon (Mj 27), un crocodile (Mj 41, Ms 74 et 75), une anguille (Ms 6, Mj 27). Tous ces derniers ont en commun la résidence aquatique et une fonction qu'il faudra préciser; ils sont et restent animaux, sans métamorphoses, et habituellement le conte ne les fait pas parler. C'est avec le héros S qu'ils entrent en rapport, apparemment sur un pied d'égalité, comme si $\mathrm{S}$ jouait le médiateur entre humains et animaux (du fait de son double aspect), entre le monde de sur-terre et le monde de sous-l'eau - celui-ci étant "inférieur", dans tous les sens du terme, et finalement vaincu.

C'est le crocodile qui revient le plus fréquemment dans la série des contes-types. Toutes ces populations lui donnent le même nom : bja (bəja). À la différence de S', le crocodile n'est pas désiré par l'héroïne, il ne lui fait pas de mal, il se borne à l'avaler (comme le ferait un python), lui sert de protection et de refuge, jamais de "mari »; en son sein, elle accouche, puis en sort vivante. Nous voyons par là que bja n'est pas le reptile carnassier de la zoologie - bien que le nom désigne aussi de vrais crocodiles - mais un « caractère » de la mythologie, un milieu habitable pour un humain qui tombe au fond des eaux.

Le serpent d'eau, la baleine et le dragon se correspondent d'un texte à l'autre ; quels que soient leurs noms particuliers, ils ne constituent qu'un même caractère, ayant la même fonction: détenteur de richesses, il doit être vaincu par le héros... lequel peut être Anguille.

41 Anguille, nduy en sré, rənuy en jörai, se situe à part. Il n'est qu'animal, il ne mue pas, mais il parle aux humains et joue l'intermédiaire entre le héros et le monde aquatique, 
bénéfique pour le petit personnage qui a confiance en lui, maléfique pour le grand personnage jaloux et peureux. Cette ambiguïté lui permet d'assumer seul les fonctions de S et S' à la fois. Quand Mj 27 le fait « devenir éléphant ", je crois qu'il ne s'agit que d'une image de sa taille énorme, d'autant plus que cet "éléphant ", va combattre sous les eaux. Quant à l'éléphant de Mj 30, il est étranger à cette série aquatique et il inverse radicalement le personnage $S$ : il est un animal sauvage qui se fait passer pour humain.

Les mythes mettent en scène quantité d'animaux parlants et d'humains à apparence animale. Pour ne retenir que cette dernière catégorie, j'ai relevé, dans la mythologie jörai seulement, tous les types de transformation qui se présentent : un homme peut se montrer sous une apparence de serpent, python, crapaud, coq, écureuil, civette, tigre ; une femme comme tigre, singe ou faisan. À cela il faut ajouter les figures de monstres : homme à oreilles d'éléphant, corps velu, jambes de cerf, etc. Ce qui est important pour notre propos c'est que, dans leur quasi-totalité, ces personnages (humains à apparence d'animal ou de monstre) changent de peau, après avoir éprouvé l'Autre, se révélant ainsi dans leur vérité humaine, et que cela se dit tuh sor, " muer ». Quelle que soit donc l'apparence, de python ou d'autre, ce qui compte c'est la transformation - et l'analyse devra s'y appuyer.

Animaux, humains, métamorphoses, ces figures risquent d'être incomprises, leur sens d'être déformé, par ceux qui feraient encore de l'homme le roi de la création, "mammifère supérieur» qui s'est donné le droit d'user et d'abuser des êtres « inférieurs »; il n'y a rien de tel dans le système de pensée des Indochinois. L'allusion à un état des choses (plutôt qu'un temps) tel que les hommes et les animaux dialoguent n'est pas tant une référence à un paradis passé, même si le discours explicite du mythe le suggère (le dit n'épuise pas la connaissance), qu'un signe d'une harmonie universelle imaginée, où l'homme n'est qu'un être parmi d'autres, dans une connivence qui rend la symbiose possible et les sociétés équilibrées.

\section{Du côté des jeunes filles, leurs noms propres}

Dans la littérature jörai, deux noms de filles reviennent souvent : H'Bia et $\mathrm{H}^{\prime} L u i^{?}$. $H$ est une particule précédant tous les noms propres de femmes. bia se retrouve en cham, c'est un titre alors : princesse, fille de sang royal (patao bia ou po bia : «la reine»). En jörai, H'Bia fonctionne comme un nom propre, très peu marqué, c'est à peine plus qu'un titre vague, connotant soit la filiation d'un seigneur (pata) local, soit la primogéniture dans une série de germains. Dans nos textes H'Bia est toujours l'aînée et n'a pas le beau rôle - l'un allant souvent avec l'autre.

lui $i^{\text {}}$ ou luc (l'occlusion glottale fermant la syllabe équivalant à une des consonnes $p, t, c$, k) signifie: fin, épuisé, extrême limite. C'est le même mot que le cham luic, qui a également cette signification; préfixé, le cham taluic désigne le «dernier né, enfant préféré, benjamin » (Aymonier et Cabaton, 1906). H'Lui? est le nom propre (mais signifiant, ce qui est généralement le cas des noms propres) de la benjamine dans les contes, où elle a le beau rôle, et parfois aussi dans la réalité. Ces deux filles étant ainsi nommées, on sait d'avance qu'elles s'opposeront, H'Lui' étant l'héroïne la plus marquée.

Dans une variante il y a une troisième sœur, plus âgée, nommée H'Kruah ; c'est le type de la vieille fille désagréable, jalouse de ses cadettes, qu'on retrouve en de nombreux textes. Par ailleurs, dans la mythologie, H'Kruah est la vieille servante du Ciel, nue et 
crasseuse ; c'est le nom que les Jörai donnent à ces tourbillons de vent ascendant qui soulèvent des colonnes de poussière. Les deux personnages sont différents, mais la H'Kruah de nos textes fait penser à l'autre et c'est voulu pour la déprécier. En fait il n'y a que deux filles, l'héroïne et l'antihérö̈ne si l'on veut ; on s'aperçoit bien que H'Kruah n'est qu'un doublet de H'Bia ; elle n'intervient d'ailleurs plus par la suite.

Dans la littérature sré le couple de sœurs est nommé Ngi et Nga, Ngi étant benjamine, donc la préférée et la préférable. Dans une variante la benjamine est nommée Nai Tölui ${ }^{?}$ et s'oppose à Nga; Ngi, citée au début, semble oubliée en cours de route. On peut en

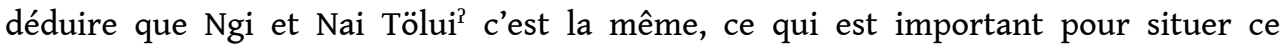
personnage qu'on retrouvera en d'autres ethnies. Nai Tölui ${ }^{3}$ est l'héroïne de plusieurs contes sré, une fois c'est une princesse cham. nai est un mot cham (et non pas sré) qui

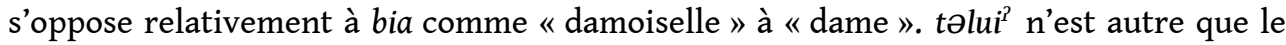
cham taluic que nous venons de voir, ou encore que le jörai lui ${ }^{2}$; Nai Tölui ${ }^{2}$ est donc l'homologue exacte de $\mathrm{H}^{\prime} \mathrm{Lui}^{2}$. Nous pouvons poser: $\mathrm{H}^{\prime} \mathrm{Lui}^{\mathrm{P}}=$ Ngi. Notons que l'ethnie sré, de langue austro-asiatique, utilise dans ses contes des noms propres cham dont la signification peut lui échapper, bien que le cham luic se retrouve en sré sous la forme luc avec le même sens.

Dans un texte ködu les deux sœurs s'appellent K'Ngi et K'Nga. $K$ est une particule précédant tous les noms propres de personnes des deux sexes; les héroïnes ont les mêmes noms qu'en sré. Dans le second texte ködu la benjamine est appelée K'Pôt ou kon Pôt ( $k J n=$ fils, fille), l'ânée n'est même pas nommée - c'est dire si elle est peu marquée. Le ködu pot (en sré : put) signifie « dernier né », «benjamin ». « On l'a appelée K'Pôt parce que c'était la dernière née » (Mk 9). K'Pôt est donc la même que H'Lui ${ }^{\text {? }}$ et que Ngi.

En böhnar le père a trois filles : l'aînée Ya Hakrôh, la puinée Bia Puön, la benjamine Bia Lui $^{2}$; ja signifie « dame ", bia « damoiselle ». En Hakrôh (röngao : Hakruah - le röngao est un sous-groupe böhnar) nous reconnaissons H'Kruah ; puən signifie " quatre », ce n'est habituellement pas un nom propre. Ces deux aînées ne font qu'un en s'opposant à la dernière ; celle-ci s'appelle Lui ${ }^{3}$, dont il est précisé kon hadruc (hatui ${ }^{?}$ en röngao), « la benjamine». Dans la deuxième partie, les filles du seigneur jaloux ne sont pas nommées ; il est seulement dit que celle qui accepte le mariage est oh hadruc, « la petite dernière ».

De cette excursion à travers les ethnies et leurs langues il ressort que les noms des héroïnes sont des traductions rigoureuses les uns des autres, désignant les mêmes personnages-types, ce qui, non seulement autorise, mais nécessite la comparaison des contextes, des fonctions remplies.

\section{Les titres des contes}

51 Mj 41a: akhan ja pum, « Conte de Mère Brousse ».

Mj 4lb: akhan ja ${ }^{2}$ don-duy hay hlui tom rit, « Conte de Mère-grand et H'Lui ${ }^{2}$ avec Rit ». Variante : akhan ala twey, "Conte de Serpent Tweng ».

Mj 115 : akhan dam tlan, « Conte de Sieur Python».

Mj 77 : akhan kra, «Conte de Singe».

Mj 50 : akhan hbia hples, «Conte de Damoiselle Fauvette».

Mj 27 : akhan rənuy-kuay, «Conte d'Anguille». 
Mj 30 : akhan ja tok-bok, "Conte de Dame Figuier » (qui a des traits communs avec Mère Brousse).

Notons qu'en jörai le conte-type, que j'ai pris comme mythe de référence, ne mentionne pas Python dans son titre; c'est peut-être dû à la place considérable que prend le personnage de Mère Brousse dans cette mythologie. En Mj 41b, comme en Mj 50, l'accent semble être mis sur la jeune fille plus que sur le héros $\mathrm{S}$ - ce qui est bien jörai, $\mathrm{S}$ n'est qu'un gendre. Ms 74 : jaljaw kla klay liay, "Conte de Kla Klang Liang » (c'est le nom de S).

Ms 75 : jaljaw trah tray lan, "Conte de Trah Trang Lan» (id.). Seul le personnage S est pris en compte, il est au centre de l'histoire.

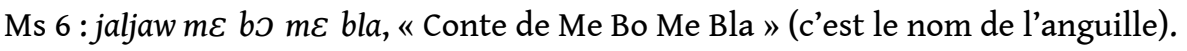

Ms 24 : jaljaw dun-wai, « Conte de Jung Wai » (nom de l'héroïne).

Ms 7 : jaljaw dul na u :r kon caw baw mo klan, «Conte d'une fille des hommes épouse d'un python ». Ici le titre est descriptif, il donne exactement l'« argument ».

Ms 9 : jaljaw kaboy, «Conte de Bông » (nom du héros S).

$\mathrm{Mb}$ : hamo :n bih klan Jعi kə bia lui ${ }^{2}$, "Conte d'un serpent python qui épousa damoiselle Lui $^{2} »$. Le titre est aussi explicite que celui de Mk 7 ; de tels titres sont exceptionnels dans cette littérature orale, au point qu'on peut se demander s'ils n'ont pas été précisés pour le collecteur, sinon composées par lui sur commentaire des informateurs.

Akhan, jaljaw et ham :n ont le même sens ; on peut les traduire indifféremment par : conte, mythe, légende. Ces populations ne font pas les mêmes distinctions que nous; par contre elles différencient, à l'intérieur de ce genre littéraire, des catégories selon le mode d'expression vocale : raconté, récité, chanté (Dournes, 1976, 329 sq. ; la typologie de ces catégories a été faite en séminaire et sera reprise dans une publication). Est akhan, etc., toute histoire traditionnelle transmise oralement, conte ou mythe, muthos.

Toutes les pièces de ce genre ont un titre ; cela permet d'en parler, sans ambiguïté, et de demander telle pièce au conteur. La plupart du temps le titre est réduit au nom propre du héros considéré comme principal - d'où l'utilité de bien noter les titres traditionnels ; c'est déjà une façon de saisir ce qui est pertinent pour telle population.

\section{Quelques formules}

Ne pouvant faire ici le relevé de toutes les «formules » des quatorze textes exploités, je me limite à quelques exemples propres à donner une idée de la nature et de la fonction de ces formules.

\begin{tabular}{|l|l|l|l|}
\hline $\begin{array}{l}y i \\
\text { Ngi }\end{array}$ & $\begin{array}{l}\text { lot } \\
\text { va }\end{array}$ & $\begin{array}{l}\text { nir } \\
\text { panier-de-pêche }\end{array}$ & $\begin{array}{l}\text { Ka } \\
\text { poisson }\end{array}$ \\
\hline $\begin{array}{l}\text { Na } \\
\text { Nga }\end{array}$ & $\begin{array}{l}\text { lot } \\
\text { va }\end{array}$ & $\begin{array}{l}\text { gir } \\
\text { pêcher-au-panier }\end{array}$ & $\begin{array}{l}\text { səs } \\
\text { serpent (Ms 74) }\end{array}$ \\
\hline $\begin{array}{l}\text { kəyi } \\
\text { Ngi }\end{array}$ & $\begin{array}{l}\text { gir } \\
\text { pêche-au-panier }\end{array}$ & $\begin{array}{l}\text { pa } \\
\text { poisson }\end{array}$ \\
\hline $\begin{array}{l}k \partial y a \\
\text { Nga }\end{array}$ & $\begin{array}{l}\text { gir } \\
\text { pêche-au-panier }\end{array}$ & $\begin{array}{l}\text { klan } \\
\text { python (Mk 7) }\end{array}$ \\
\hline
\end{tabular}



boire, etc.), si c'est une fille j'en ferai mon amie, si c'est un garçon j'en ferai mon mari (ou mon gendre)» - avec des variantes, notamment si le sujet parlant est un homme, cas plus rare. On pourrait appeler cette formule toute faite, qui revient plus fréquemment dans la littérature, l'Engagement imprudent (cf. Mj 30, 41b, 77, 115). Elle vient habituellement au début du récit et entraîne toute la suite, avec des conséquences pour le sujet parlant ou pour sa fille, c'est-à-dire un mariage insolite. Elle trouve, en quelque sorte, sa contrepartie dans la «formule rassurante " (qui est aussi une forme d'épreuve) : "Si tu m'écoutes, je t'aide » (Mj 30) ou "Ne crains pas, n'aie pas peur " (Mj 27, Ms 6). À la formule de promesse inconsidérée émanant de la jeune fille «paumée » en forêt, correspond la parole de Mère Brousse qui, elle, sait très bien ce qu'elle fait, car elle tient du «village » et du "sauvage », entre société et forêt; de par cette situation, sa parole rejoint celle de Dame Figuier, rassurante pour celle qui aura confiance en elle. Ces paroles-formules sont des avertissements pour le héros (l'héroïne) et pour l'auditeur du conte, sur des registres différents. La logique de cette mythologie consiste en un réseau de relations entre des caractères dont il convient de reconnaître les correspondances profondes dans le contexte des situations décrites.

2. "Si on t'invite à sortir (à aller voir des coqs, des buffles se battre, etc.) n'y va pas, reste à la maison!» (quelquefois même : "Ne parle pas avec des gens de l'extérieur »). C'est la parole qu'adresse à sa femme le mari sur le point de s'absenter (Mj 41a et b, 77, 115 et aussi Ms 75) toujours pour se rendre sur la côte orientale. Plus qu'une recommandation de prudence manifestant quelque pressentiment chez l'époux, c'est un véritable interdit, avec toute la force que la notion peut avoir dans ces sociétés. La formule Interdit en entraîne habituellement une autre, la Prémonition : au loin, le mari sent sa bague glisser de son doigt, par exemple, et il dit alors : « Malheur est arrivé à ma femme ", ce qui le fait revenir à la maison. Le récit s'articule sur ces paroles fortes. La formule d'interdit (présente dans la séquence 10 des résumés), comme parole adressée à l'épouse, ne se retrouve $\left(n^{\circ} 10^{\prime}\right)$ chez les Austroasiatiques que dans un 
contexte modifié, sauf en Ms 75 qui, par la présence de 10 et 10' à la fois, établit un pont entre les textes austronésiens et austro-asiatiques. En Ms 75, Mk 7, Mk 9, l'interdit prononcé par le héros vise sa belle-sœur (l'aînée jalouse) - en Mk 7 exactement dans les mêmes termes qu'en Mj 41b. Comme dans le premier cas, l'interdit est violé ; mais cette fois-ci, l' « héroïne en négatif » n'en sortira pas vivante, bien que, à ce moment-là, elle n'ait pas été plus «coupable » que sa sœur - elle a agi par inadvertance. Il nous faut comparer les contextes pour interpréter cette différence. Dans les versions jörai et sré du conte-type, l'héroïne cadette se tire d'affaire sans l'intervention du mari ; dans les versions sré et ködu l'aînée a été sauvée une première fois par le beau-frère, elle ne peut l'être une seconde fois; ce n'est pas seulement sa jalousie et son égoïsme qui l'opposent à la cadette, mais aussi le fait qu'elle ne peut rien réaliser par elle-même. Dans la société jörai (et secondairement chez les Sré), la femme sait, mieux qu'ailleurs, se passer de l'homme.

Inversement l'homme exprime sa façon propre de se passer de l'épouse - sinon de la femme. Dans des mythes de chasse jörai, on retrouve la même formule d'interdit : avant d'aller poser ses pièges, le chasseur dit à son épouse: «Ne sors pas de la maison, n'adresse la parole à personne de l'extérieur !» : grâce à cela les divinités féminines de la forêt souriront au chasseur en conduisant les animaux vers ses pièges. Si la femme viole l'interdit, ce n'est pas elle qui en pâtira, c'est le chasseur, qui reviendra bredouille ; il n'aura pas perdu que le gibier, mais encore l'amitié des filles-forêt. Cette similitude de formules (dans leur nature et leur fonction) nous invite à rapprocher le voyage de $\mathrm{S}$ d'une expédition de chasse et aide à comprendre que la violation de l'interdit perturbe plus le mari que la femme (même dans son crocodile, l'héroïne n'est pas inquiète). Parce que le même interdit n'a pas été respecté, le chasseur perd les dons des nymphes et le héros $\mathrm{S}$ perd sa femme - pas plus la maison que le sein du monstre aquatique n’a pu retenir celle-ci, jamais piégée finalement.

\section{Des énoncés}

Ici encore je ne prendrai que quelques exemples pour illustrer le fonctionnement de la structure mythique.

66 1. Énoncé de départ (ou incipit). Alors que les énoncés d'arrivée (ou d'aboutissement) de ce conte-type (et de tant d'autres) n'ont guère plus d'originalité que le cliché « et ils vécurent heureux ensemble ", quand ils sont exprimés (il est rare que le conteur aille jusqu'au bout, si tant est que le mythe en ait un, soit que le texte s'étende trop, soit que l'assistance s'endorme, soit que le récitant se lasse avant), l'énoncé de départ décrit la situation originelle, entraîne toute la suite, comme un titre développé, et attire l'attention.

67 L'incipit du conte-type jörai présente Mère Brousse qui va chercher des ignames en forêt; nous sommes donc en régime de cueillette, très probablement en saison des pluies (vers septembre-octobre) ; une averse lui fait rencontrer S. L'énoncé initial de Ms 74 décrit une situation de pêche, celui de Ms 75 évoque la chasse, toujours occasion de rencontrer $\mathrm{S}$. De même $\mathrm{Mk} 7$ et aussi $\mathrm{Mb}$, l'arbalète servant essentiellement à la chasse. Tous ces débuts se réfèrent aux procédés archaïques (et encore actuels en période de disette) d'obtention de la nourriture, par des peuples aujourd'hui cultivateurs. La différence principale consiste en la personne qui s'y livre : une vieille femme chez les austronésiens jörai, des hommes (le père ou un frère aîné) chez tous les 
autres, qui sont austro-asiatiques. Ce qu'il fallait c'était faire démarrer l'histoire dans un milieu entre nature et culture : le personnage-déclencheur (humain) cherche une nourriture sauvage et rencontre le héros qui se manifeste comme animal et se révèle ensuite un humain, en vérité et non plus en apparence. L'homme (père, plus rarement frère aîné) s'engage plus ou moins inconsciemment en prenant des risques pour sa fille (sa sœur) ; la vieille dame, apparemment irresponsable, sait bien ce qu'elle fait, car ellemême tient du monde des humains et de celui de la forêt, de la réalité et de la féérie, des deux côtés du miroir.

2. Enchaînements et déplacements d'énoncés (pris dans des séquences). Dans le texte retenu comme mythe de référence, $\mathrm{Mj} 41 \mathrm{a}$, j'ai numéroté des séquences selon leur succession dans ce conte-type; ces séquences, abstraites pour le travail présent, englobent des formules et des énoncés, sans les exprimer tous, bien entendu. Ce sont des schémas provisoirement suffisants pour que nous observions leur découpage et le comparions d'une version à l'autre.

Les numéros 1 (qui comporte la formule «Engagement imprudent» et l'énoncé de départ), 2, 3 et 4, se trouvent dans cet ordre, à peu de choses près, dans toutes les versions du conte-type, des Sré aux Böhnar en passant par les Jörai. C'est l'ensemble le plus stable, qui ne peut se déliter ; 1 étant posé, on enchaîne nécessairement jusqu'à 4 . De même 6,7 et 8 , là où ils sont présents, paraissent toujours dans cet ordre et d'un seul tenant; mais cet ensemble peut être séparé du premier (1-4) par un paquet de séquences tel que $10-13,5$ et 9 étant quasi facultatifs. Quand paraissent les séquences 10 '-11' elles suivent nécessairement l'ensemble 6-8, quelle que soit la place de celui-ci. La finale 14-15 n'est présente qu'en jörai et en sré ; pour qu'elle apparaisse il faut qu'elle ait été précédée par 9 (quelle que soit la place de 9, du moment qu'il est antérieur).

70 L'ensemble 1-4 $( \pm 2)$ apparaît sept fois, l'ensemble 6-7 $( \pm 8)$ six fois, l'ensemble 10-13 ( \pm 10) quatre fois, $10^{\prime}-11$ ' trois fois. Cette sèche statistique a l'avantage de répartir les ensembles selon leur fréquence d'apparition et de montrer par conséquent, à travers diverses traditions, lesquels sont les plus stables.

71 Ces exemples permettent de constater deux faits : a) l'existence de suites (ou ensembles partiels) qui constituent un tout mémorisé, b) la variabilité de leur ordre de succession - comme s'il fallait tout dire sans trop s'occuper de la place relative des éléments. Il me semble que ces deux faits s'articulent sur deux opérations de la mémoire; il y a une mémoire, sinon passive moins consciente, qui enregistre un donné relativement continu, et une mémoire de «rejeu» (rappel), plus active (ou plus consciente); cette distinction recoupe à peu près celle que l'on devrait faire entre "se souvenir » et "se rappeler ». N'oublions pas qu'il s'agit de littérature orale et que tout le dit a été d'abord entendu, puis mémorisé. Les paquets indivisibles de séquences sont des parties de ce tout culturel enregistré, leur succession (plus libre) est un phénomène de rejeu. Les ensembles stables figurent ce qui se transmet, pour tous, dans une culture commune ; les enchaînements variables représentent ce que la pensée (plus individuelle, propre au conteur ou à une traduction locale) associe - sans oublier de tenir compte de ces accrochages de formules ou d'énoncés qui sont dus à des phénomènes quasi mécaniques d'enchaînement par le son (assonance) ou par le sens (annomination). Entre ces ensembles stables et l'ordre variable de leur succession on pourrait voir une différence comparable à celle qui existe entre vocabulaire et syntaxe, ce qui donne à réfléchir quand il s'agit de langues à syntaxe de position : le sens change relativement à 
l'ordre respectif des éléments. Toutes les versions ont-elles le même sens dans toutes les ethnies où elles ont été relevées? Ce n'est pas évident. Jusqu'à présent nous avons vu comment le mythe est fabriqué et comment il signifie, il nous restera à chercher ce qu'il signifie.

\section{Du côté des Cham}

72 J'ai relevé plus haut des vocables qui se retrouvent dans la langue cham, ainsi : bia "dame ", nai « damoiselle », taluic «benjamine ». Pour ne pas parler du jörai qui, de famille linguistique austronésienne, est très proche du cham, c'est le ködu, parmi les austroasiatiques (notamment Mk7), qui est le plus riche en termes cham. Ce phénomène n'est pas étonnant quand on connaît la situation des Ködu. Le groupe ethnolinguistique churu de la vallée de $\mathrm{Da}^{{ }} \mathrm{Nying}$ est divisé en deux sous-groupes : l'un austro-asiatique, habituellement appelé ködu, l'autre austronésien, ou churu proprement dit, plus proche du cham que le jörai lui-même. Le type d'habitation (sur pilotis chez les Churu, à ras du sol chez les Ködu) et la localisation géographique (villages homogènes churu ou ködu, en rive gauche ou en rive droite de la rivière) ne différent pas au point qu'il n'y ait pas une certaine imbrication ; une bonne proportion des hommes est bilingue. Ainsi s'explique la présence de termes purement cham dans des textes ködu (austro-asiatiques), notamment adat, "la coutume», mot malais inconnu en jörai, mathir, "palais royal », qui se retrouve en jörai sous la forme athir, «siège" (matériel rituel pour le «Roi» du Feu). Un peu d'histoire pourra faire comprendre ce qui s'est passé.

73 À l'époque de l'apogée d'Angkor (XIII ${ }^{\mathrm{e}}$ siècle) la péninsule indochinoise était partagée entre deux grands empires hindouisés : le Kampuchea (Cambodge en français) à l'ouest, le Champa à l'est; entre deux, tampon mais non État, les minorités autochtones. En ce temps-là les Böhnar et surtout les Jörai étaient plus ou moins inclus dans l'empire cham. La guerre de conquête des Viêt, venus du nord, repoussa peu à peu les Cham vers le sud; après le xvII ${ }^{\mathrm{e}}$ siècle, les Cham n'occupaient plus qu'une parcelle de côte au sudest des Churu et Sré, non sans avoir influencé ceux-ci (dans la langue et les techniques). Chez les Böhnar et les Jörai on trouve des ruines de monuments cham en pierres et en briques, vestiges de la grande époque; chez les Churu-Ködu sont conservés des " trésors " cham, objets royaux de la décadence gardés dans des huttes en bambou et paille. Quelle que soit la date à laquelle elle ait commencé, la chamisation des Churu s'est prolongée plus tardivement que celle des Jörai, d'où ces traces bien vivantes dans la littérature orale des Churu-Ködu d'aujourd'hui.

Vu l'impact de la culture cham sur les quatre ethnies dont des textes sont présentés ici et les marques linguistiques qu'elle y a laissées, on peut se demander si notre contetype à quatre voix n'a pas un correspondant dans la littérature cham. Celle-ci est encore très peu connue ; toutefois un conte recueilli (Landes, 1887) mérite attention.

« Noix de coco » n'est qu'une tête sans corps. Il advient qu'il garde les buffles du roi. H’Bia, la fille benjamine de celui-ci, apporte tous les jours son riz à « Noix de coco ». Débarrassé de son enveloppe, le héros se révèle être un beau jeune homme, H’Bia l'épouse. Les deux sœurs aînées de H'Bia, la princesse, jalousent leur cadette ; H'Bia est noyée. Recueillie dans un coquillage, elle atterrit sur la grève, est hébergée par un couple de vieux. Elle fait un beau tissage, dans lequel elle a introduit la bague de « Noix de coco»; les vieux portent ce tissage au roi, qui reconnaît la bague de son gendre. « Noix de coco » va rechercher sa belle. 
Si fragmentaire que soit l'information, elle se révèle d'une grande utilité. La transformation du héros, la jalousie des sœurs aînées qui noient la benjamine - et jusqu'au détail du coquillage - sont autant d'indices d'un fonds commun à toutes ces histoires. Le héros «Tête unique » a un équivalent dans la littérature jörai et, par là, rejoint ces figures de monstres (transformables) épousés par une princesse. Comme je l'avais signalé, l'essentiel n'est pas qu'il y ait un serpent, mais une mue.

Le conte cham est-il un écho ou une source des textes indochinois? Je formule provisoirement l'hypothèse qu'il s'agit plutôt d'une souche unique, pré- indianisée, que les Cham (des Jörai hindouisés) auraient récupérée à leur façon, d'autant plus qu'il n'y a aucune marque d'hindouisme dans ce conte cham. Nous nous retrouverions donc au stade ancien d'une culture commune, sans rien pouvoir suggérer de son origine.

\section{Une analyse possible}

77 J'estime qu'on ne peut se dispenser de la démarche suivie jusqu'ici, si l'on prétend chercher des sens d'un mythe dans un ensemble : sens qu'il a pour ceux qui le récitent et l'écoutent, sens qu'il peut avoir pour ceux qui l'analysent, à travers et par delà les spécificités culturelles. Avant d'en venir là, je ferai état d'une impasse comparatiste.

78 L'image du serpent est attestée dans des mythologies très différentes et éloignées les unes des autres. Dans la Bible, dès la Genèse, le serpent est l'Ennemi de Dieu ; le trait est souligné chez les Prophètes; "Yahweh sévira.../contre Léviathan le serpent fuyard/ contre Léviathan le serpent tortueux/ et il tuera le dragon qui est dans la mer » (Isaïe, $21,7)$; le serpent habite les eaux profondes (Amos, 9, 3) - ce qui rejoint la mythologie phénicienne - et il est parfois nommé Léviathan, qui peut être dragon, monstre marin, crocodile (Job, 40, 25). Pas de connotation positive dans le monde biblique, sinon l'ambigu Serpent d'airain (Nombres, 21, 8), signe de guérison, qu'on retrouve sur le bâton d'Asklépios (différent du caducée de Mercure). À l'opposé, le dragon, comme emblème-type de la culture chinoise, est une puissance bénéfique (à condition de ne pas être mécontentée), une force de la nature; plus proche du pays de nos contes, le Vietnam a hérité de la Chine sa conception du dragon, qui en faisait une figure de l'empereur ; dans la croyance populaire rồng est un génie chtonien, rejoignant ainsi, sur ce point entre autres, la pensée des autochtones indochinois. Cela peut aider à situer le dragon de leur mythologie, cəgrai (cham : nagarai, sanskrit nāga rāja), entre l'Inde et la Chine, mais ne nous dit rien sur sa fonction de monstre aquatique qu'il est ici; cela nous éclaire encore moins sur la signification du personnage Serpent - notons toutefois une constante : dragon est associé à richesse, et ce jusqu'à nos jours et en Occident (Tolkien, 1949).

79 Par le précédent numéro des Cahiers de littérature orale (CLO, 1: 115) nous avons rencontré un serpent lithuanien qui extorque une promesse de mariage à une jeune fille, l'entraîne au fond des eaux et lui fait des enfants. Nous ne savons pas grand-chose de plus de ce personnage $S$, sinon que, dans d'autres versions, il peut être remplacé par un succube ou par le diable. Voilà qui paraît se rapprocher de notre conte; en fait il ne s'agit que de ressemblances superficielles et les significations sont bien différentes.

On pourrait faire ainsi le tour de toutes les mythologies du monde, évoquer Mélusine changée en serpent dans la partie inférieure de son corps - ce qui fait penser à Mk 7 $\mathrm{n}^{\mathrm{o}} 4-$, ce serait aussi gratuit. 
81 Je m'arrêterai un peu plus sur Psyché. Un roi a trois filles; la benjamine, richement parée (Ms 75, n 3), est conduite vers une montagne pour être mariée à un « serpent »en fait c'est Éros qui, invisible, emmène Psyché dans un palais. Les sœurs aînées, jalouses, veulent la perte de leur cadette - celle-ci devra faire un stage aux enfers pour renaître et vivre avec Amour sur l'Olympe - les aînées veulent posséder Éros, mais ne réussissent qu'à se tuer. Il est certes aisé de relever les points communs avec notre conte-type, mais il n'y a pas de serpent dans cette histoire, pas même en apparence on a seulement fait croire à la jeune fille, sur la foi de l'oracle, qu'elle allait épouser un serpent. B. Bettelheim (1976 : 357 sq.) fait une étude intéressante de ce mythe, qu'il place dans le « cycle du fiancé-animal » dont le type parfait est «La Belle et la Bête ». Notons tout de suite que, dans ce cycle occidental, la Bête n'a telle apparence qu'en punition d'une faute ou par la malveillance d'une sorcière; le héros "Bête" indochinois dispose à son gré de son apparence et en change à plaisir. La différence est fondamentale.

Dans son optique occidentale et freudienne Bettelheim base son interprétation sur les phantasmes (qu'il prend à son compte) d'une certaine société européenne : le sexe est animal (1976, 349, 350, 351 - il se répète), la jeune fille a des angoisses sexuelles (1976, $344,354,364)$, mais le méchant serpent phallique repoussant (= le mâle) se montre dans sa vérité de beau jeune homme et la jeune fille, d'abord oedipiennement attachée à son père, finit par apprécier cet amour. Ainsi se débarrasse-t-on de ses complexes. Si un Jörai entendait cela et y comprenait quelque chose, il y verrait peut-être la marque d'un profond trouble mental. Dans la vie le sexe est attirant et la jeune fille se réjouit de faire l'amour, c'est bien " naturel »; dans le mythe c'est le père qui est angoissé pour sa fille, mais celle-ci va au "serpent » en toute confiance - et même dans le mythe de référence ( $\mathrm{Mj} 4 \mathrm{l})$ la mère-grand, quasi omnisciente, a la maîtrise des événements - ; si la sœur aînée refuse, ce n'est pas par angoisse sexuelle, mais tout simplement parce que, ne sachant ni rêver, ni imaginer, ni aimer, elle se croit devant une bête ordinaire. L'interprétation psychanalytique s'écroule, pour cette société du moins.

Des images, apparemment semblables, peuvent avoir des sens totalement différents selon les cultures. Nous ne sortirions de l'impasse comparatiste que si nous cherchions à voir, sur une grande échelle, comment ces différences peuvent s'organiser. J.R.R. Tolkien (1964, 22-23) dit de certains comparatistes qu'ils sont inclinés à déclarer que deux histoires, bâties autour du même motif, sont «la même histoire »; il continue : « It is the coulouring, the atmosphere, the unclassifiable individual details of a story, and above all the general purport that informs with life the undissected bones of the plot, that really count »-c'est exactement ce que je pense et essaie de prouver.

Si on tente un classement selon les thèmes ou les motifs, où va-t-on placer notre conte indochinois ? Le fiancé-animal ? Les deux sœurs ? La victoire du cadet ?... Tout choix de ce type risquerait fort d'être une interprétation ethnocentrée, sinon une distorsion, en laissant échapper les neuf dixièmes du dis cours pertinent. Avant de chercher ce qui est « pareil» (travail facile et superficiel) pour étiqueter commodément, il me semble plus important d'étudier d'abord l' « inclassifiable " pour voir ce que des traditions diverses font d'images plus universelles et, au sein de la culture qui nous retient, « rapprocher les versions différentes en vertu de leurs 'différences'« (Detienne, 1977, 24) pour découvrir un sens dans leur ordonnance.

Préparée par une expérience incomplète en pays sré, la longue pratique de la mythologie jörai m'a montré qu'une cause de différences dans les variantes peut se 
trouver dans la personne du conteur, selon son âge notamment ou l'étendue de sa culture (ce qui n'est pas toujours lié), sans oublier que ce conteur reste dépendant d'une tradition locale (parfois limitée à un village ou une famille). J'ai constaté ce phénomène sur un très grand nombre de textes, surtout quand tel mythe précis m'a été raconté une première fois par un jeune garçon ou une jeune fille, et que je l'ai réentendu récité au village par un ou une spécialiste. $\mathrm{Si}$, dans les deux cas, l'histoire peut se résumer de la même façon, se réduire à une trame unique, la signification n'est souvent pas la même. On raconte l'histoire de telle manière parce qu'on l'entend ainsi ; toute performance révèle l'intelligence d'un sens, à tel point que, sur une quantité d'exemples, il est possible de distinguer des niveaux de signification, réels dans la société envisagée. Je l'ai éprouvé dans l'intimité des divers milieux, dans leur relation avec la littérature orale : les individus très jeunes, les gens du commun, des personnes plus cultivées (connaissant notamment plusieurs textes longs).

Je donne dès maintenant ce tableau synoptique (ci-contre) pour le rôle des conteurs ; j'aurai à y revenir par la suite. Les versions sré, recueillies quinze ans avant les autres, ne portent pas de nom d'exécutant. Les deux variantes jörai du mythe de référence proviennent de deux dames d'un certain âge, du même village; elles reflètent une tradition unique. La conteuse de $\mathrm{Mj}$ 4lb (grand-mère de $\mathrm{H}$ 'Er) a une connaissance plus étendue que celle de 4la (H'Kreng) ; à son programme figurent une histoire où apparaît un dragon et une autre dont le héros est un homme d'apparence monstrueuse (non animale); l'absence du personnage $S$ ' (cas unique) dans la variante qui lui est propre n'est pas un manque : c'est S qui compte, il suffit au sens. Du conteur de Mj 115 (père de Bhung) j'ai entendu quatre longs récits.

Les conteurs de Mk 7 (père de Tuyen) et de Mk 9 (père de K'Té) appartiennent à des sous-ethnies différentes; celui de Mk 9 a à son programme plusieurs textes longs, dont un mythe d'origine ; il est le seul à présenter le héros $\mathrm{S}$ d'abord comme jeune homme ; ainsi se trouve soulignée la liberté du héros qu'on voit prendre une apparence à son gré - ici celle d'un dragon, ce n'est donc pas le serpent en tant que tel qui compte, mais le jeu des transformations.

Dans l'ensemble toutes les versions proviennent de personnes d'âge mûr et assez cultivées. Sur l'exemple d'autres contes recueillis dans leur version " enfantine » (c'està-dire contés par un enfant, et non pour des enfants), dont le héros est un être plus ou moins monstrueux qui se transforme, il est possible de prédire le sens immédiat que le public jeune donnerait à notre série « Serpent ». Jamais je n'ai vu d'enfants, même très jeunes, terrorisés par la description de ces monstres; s'il s'agit, par exemple, d'un dragon maléfique, ils se réjouissent de le voir vaincu; si c'est un héros bénéfique, ils exultent de le voir se transformer et berner les puissants - et tout cela ils le savent d'avance. Le plaisir consiste en l'audition d'une histoire bien dite dont le sens premier répond à leurs désirs: victoire des méprisés et des benjamins sur des "grandes personnes » qui ne voient que les apparences. 




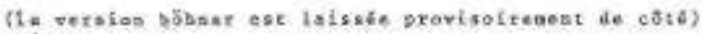
explicetion des signes I

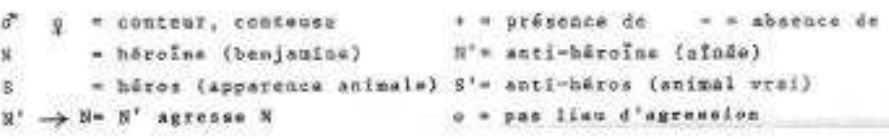

89 Les intonations, la scansion par des formules typiques, certaines insistances, des questions posées par l'auditoire interrompant le conteur, des commentaires subséquents (beaucoup plus rares) sont autant d'indications sur des significations au niveau de l'auditeur plus cultivé, mieux au courant d'une partie (sinon de l'ensemble) de sa mythologie - ces significations composant avec le sens premier, dégagé plus haut, mais ne l'excluant pas ; le sens s'épaissit, à chaque niveau, comme une bougie plusieurs fois replongée dans un bain de cire. Le personnage du héros à transformations (mues) successives étant archiconnu de tous, l'opposition des deux sœurs fait saillie sur un thème commun. À ce sujet l'auditeur moyen notera tout de suite une anomalie: la cadette est mariée avant que l'aînée ne le soit ; ce qui serait inconvenant dans la réalité sociale devient signe dans le mythe (qui ne calque pas, loin de là, le vécu quotidien). La benjamine est classiquement la préférée et c'est elle qui doit réussir. Au départ il y a deux filles, à l'arrivée il n'y en a plus qu'une : l'héroïne heureuse avec $\mathrm{S}$. Tout se passe comme s'il fallait en éliminer une des deux; apparemment ce devrait être la cadette, donnée au serpent, en fait ce sera l'aînée qui a manqué cette occasion qu'on ne retrouve pas deux fois. N' ne paraît que pour mettre en valeur le rôle de N. Il ne s'agit là plus tant de deux individus différents que de deux aspects d'un seul personnage ou de deux possibilités entre lesquelles il a à choisir. Un tel dédoublement, souligné par le parallélisme des noms propres (H'Lui ${ }^{2}$ et H’Bia sont culturellement aussi couplés que Ngi et Nga), est familier à ces Indochinois connaisseurs de leur mythologie, que le second membre de la paire soit le reflet (l'écho) ou l'opposé du premier.

$S$ et S' ne seraient-ils pas dans le même rapport? Le point de vue du chasseur (le spécialiste, cultivé aussi dans tous les domaines) est intéressant à cet égard : l'animal (surtout de grande taille) rencontré en forêt, peut être autre chose qu'un simple animal ; on n'en est jamais sûr ; il est toujours ambigu, en relation avec les divinités sylvestres, son image est associée à celle de héros mythiques. Le chasseur lui parle, le ménage parfois. Mythes de chasse et propos de chasseurs me l'ont appris (et rappelonsnous les interdits de chasse signalés plus haut). Dès lors nous sommes sur la voie d'une signification de la paire S-S': ce personnage ambigu pourrait être $S$ et $S^{\prime}$ selon la personne à laquelle il s'adresse - et n'oublions pas qu'il fut un temps où animaux et végétaux conversaient habituellement avec l'homme. S' existerait alors comme pour 
montrer ce que S est, n'est pas, ou pourrait être, selon les cas. Quoi qu'il en semble, le système de pensée des ces populations du Sud-est asiatique n'est pas dualiste, il ne s'arrête pas à de rigoureuses oppositions binaires, sa passion du continu lui fait partout chercher le moyen terme - l'analyse de taxonomies en fournit des exemples (Dournes, 1971).

91 Le chasseur va et vient entre le village et la forêt, il a des accointances dans les deux milieux. Le paroxysme de cette ambiguïté est vécu par ces hommes «anormaux » qui mènent une double vie : au village, avec leur épouse, ils font comme tout le monde, lors de fugues en forêt ils se dépouillent de tout vêtement, parlent avec les fauves, font l'amour avec des filles-forêt. Ce dédoublement du moi n'est pas sans analogie avec le double état de $\mathrm{S}$, mais le possédé visionnaire ne sait plus qui il est, à la différence de $\mathrm{S}$ maitre de la situation. Le chasseur sait qu'il risque d'aller trop loin dans le milieu sylvestre et d'y perdre son identité, il ne peut manquer de rapprocher $\mathrm{S}$ et le possédé, sans les confondre, puisque $\mathrm{S}$ réussit en résolvant la dualité.

Les connaisseurs de leur mythologie sont habitués à des transformations : par travesti ou mue (le plus souvent en milieu forestier), par mort et résurrection (presque toujours en milieu aquatique). Quand, dans la même histoire, c'est un jeune homme qui mue sur terre et une jeune fille qui ressuscite $\mathrm{du}$ fond des eaux, ces auditeurs avertis sont alors certains d'avance de l'issue : les jeunes gens feront un couple heureux - comme si la vie à deux nécessitait une refonte totale pour naître à un état nouveau - à l'inverse de S' qui ne se transforme pas et de N' qui ne renaît pas. Dans les cycles parallèles de Drit et de Diung il arrive que le héros doive passer par une cuisson : la vieille Mère Brousse le découpe, le met dans un chaudron ; quand il est bien cuit, elle remodèle un être neuf. Le feu (qui est l'équivalent de la mue) est alors au jeune homme ce que l'eau est à la jeune fille. Par dizaines les textes illustrent ces processus, au point qu'on peut les dire systématiques. De plus il est fréquent que la mue du garçon soit jetée au fil de l'eau ; à ce moment se présente une autre image : le rite de réparation de l'inceste après l'union constatée de deux jeunes gens de même clan (si éloigné soit leur cousinage). Les fautifs sont conduits au bord de l'eau, ils y descendent, là se dépouillent de tout vêtement (ceinture pour le garçon, jupe pour la fille) qu'ils jettent au fil de l'eau, vers l'aval et la perdition, puis remontent tout nus et tout neufs, comme des êtres devenus différents ; ils peuvent alors vivre ensemble.

93 Je précise que tous les éléments qui ont servi à cette première étape de mon analyse sont le fruit d'une longue intimité avec des Jörai surtout et de conversations suivies avec des hommes si intéressés par leur propre culture qu'ils en sont venus à recueillir eux-mêmes des mythes et à en faire la transcription dactylographiée. Quand on a cette possibilité de connaître un tel contexte ethnographique vivant, on aurait tort de ne pas s'intéresser d'abord au sens qu'une population donne à ses dits traditionnels, ou explicites dans un discours spontané avec un ami de passage. Et c'est à partir de là et en profitant (faudrait-il s'excuser d'être un profiteur en la matière ?) des différentes versions dans les autres ethnies (dont aucun membre n'a actuellement ma possibilité de disposer de toutes les versions à la fois) que je vais affiner l'analyse - travail plus personnel et, par là, plus aléatoire.

Revenons d'abord au tableau synoptique (cf. supra : 97) pour voir comment, sur un fond de tissu commun, les différences peuvent s'organiser. En comparant Mj 41a et $41 \mathrm{~b}$ à Mk 7 et 9 nous constatons que « mort de $\mathrm{N}$ » est lié à « relation d'agression N' $\rightarrow \mathrm{N}$ " ", ce qui est bien normal puisque c'est $\mathrm{N}$ ' qui cause la mort de $\mathrm{N}$; quand cette relation 
d'agression manque, il n'y a pas de mort de N, du moins sous cette forme criminelle. On peut se demander, en tenant compte de l'ensemble mythologique, si la mort de $\mathrm{N}$ ne doit pas être supposée parce qu'elle serait nécessaire. Reprenons le mythe de référence Mj 41a; nous y relevons deux «morts» de N : l'une de forme classique, au fond des eaux, par la faute de $\mathrm{N}^{\prime}\left(\mathrm{n}^{\circ} 12\right)$, l'autre, qui la précède $\left(\mathrm{n}^{\circ} 3\right)$, dans l'eau aussi, quand $\mathrm{N}$ veut rattraper la dépouille de $\mathrm{S}$. Cette séquence est un cliché de la mythologie, elle revient presque chaque fois que l'héroïne va se lier à un être à transformations; dans ce contexte elle est présente à la mémoire de tous, à tel point que le conteur puisse la sous-entendre sans l'exprimer. Cela pourrait expliquer son absence des versions ködu. Même si N' ne provoque pas la mort de N, N doit renaître de l'eau.

Le rapprochement de Mj 115 et Ms 24 apporte une lumière nouvelle : dans ces deux seuls cas N' est absente, $\mathrm{N}$ ne meurt pas mais disparaît, enlevée par des seigneurs ennemis (qui ont nom Fourmi, Termite...) ou quasi enlevée par un seigneur des eaux, pendant que son mari était en voyage ; celui-ci doit ensuite reconquérir son épouse, la faire revenir à la vie normale, ce qui équivaut à une renaissance. Je ne développe pas ce point qui nous entraînerait sur de nouvelles pistes et attirerait bien d'autres textes encore.

J'avais laissé de côté la version böhnar; malgré l'identité apparente de thème, elle est très différente de structure et, par là, de sens. La première partie du récit pourrait se suffire à elle-même, la seconde partie n'en est que le négatif, la relation N'-S' inversant $\mathrm{N}-\mathrm{S}$, en miroir, comme pour montrer ce que cela aurait pu donner si S n'avait été que serpent - ce qui n'est pas sans éclairer la fonction du couple N'-S' dans les autres versions ; mais, à mon avis, ce n'est pas exactement la même histoire, ne serait-ce que pour le rôle essentiel du père comme je l'ai déjà signalé. Qu'il s'agisse de filles de familles distinctes au lieu de sœurs me semble constituer un de ces cas où les différences se ressemblent. En revanche la version böhnar est beaucoup plus proche de deux textes khmer (du second surtout) que Solange Thierry me communique ${ }^{5}$ : même structure en miroir inversant, même rôle primordial des parents. En somme, des Cham aux Khmers, en passant par les minorités ethniques de l'hinterland, un même canevas se présente : début en forêt (l'Arbre y est souvent du genre Ficus - banian ou autre -dre en ködu, drai en khmer), fin dans l'eau, avec un héros « serpent » divinement beau ; sur ce canevas chaque peuple a brodé son histoire, telle qu'il l'entendait. Il est probable que d'autres textes viendront enrichir ce corpus sud-est asiatique, ce qui pourrait amener à discuter l'analyse que je propose.

En attendant je me limite aux versions retenues sur mon tableau et j'en viens à ce que j'ai appelé les "milieux signifiants", soit Arbre et Eau (présents également dans les versions böhnar et khmer, mais je ne les commente que dans les contextes ethnographiques que je connais le mieux). L'arbre, où Python se trouve lové, est le plus souvent un banian, de taille élevée et de frondaison épaisse, symbole de fécondité et de richesse; de milieu sylvestre il a des connotations plutôt ouraniennes (en Ms $75 \mathrm{~S}$ est petit-fils de Soleil, la Dame du Figuier de Mj 30 est actuellement sur la Lune). L'eau est toujours une nappe (en mouvement ou non), rivière, étang ou océan. Un ami jörai m'expliquait : « Une surface d'eau est comme une femme, la pluie sur l'étang est comme l'homme qui vient la féconder "-composant ainsi l'horizontalité et la verticalité. À partir de telles conversations et de déductions on pourrait dresser un schéma de ce type, à lire comme des séries de correspondances (colonnes) qui se répondent plus qu'elles ne s'opposent terme à terme : 


\begin{tabular}{|l|l|}
\hline arbre & Eau \\
haut & bas \\
ouranien & chtonien \\
amont & aval \\
est & ouest \\
vertical & horizontal \\
terre & mer \\
solide & liquide \\
hauteurs & gouffre \\
homme & femme \\
python(tlan) - S & serpent (ala, bəs) d'eau - S' \\
homme à transformations & crocodile, dragon, baleine \\
homme dehors & femme dedans \\
instable & stable \\
\hline
\end{tabular}

Dans les sociétés matrilinéaires que j'étudie, la place de la femme est dans la maison, elle ne sort du village que pour aller au champ voisin, au bois ou à l'eau. L'homme quitte son village pour venir habiter chez son épouse ; rarement dans la maison, il sort beaucoup ; toujours en mouvement, il fait le lien entre son milieu et celui de sa femme ; les héros de la mythologie font de grands voyages au loin, les guerriers s'affrontent très haut dans les airs, dans la région des nuages. Ces quelques précisions montrent que la simplification du schéma ne doit pas faire penser à des paires d'un rigoureux système binaire; entre les deux colonnes il y a un mouvement, médiation, et c'est notamment la fonction de S : il est en forêt comme chez lui, il commande jusqu'au fond des eaux, il relie tous les milieux (déplacement de sa mue d'amont en aval) ; son polymorphisme lui permet d'entrer en rapport avec les espèces sauvages aussi bien qu'avec les hommes ordinaires.

Le rapprochement de Ms 24 et Mj 41 montre une similitude des fonctions de S' et Crocodile; S' est du côté de ces animaux aquatiques, pas nécessairement maléfiques, qui peuvent avaler une femme et la restituer vivante ou ressuscitable. Reportons cela sur les mythe de référence où $S$ ' et Crocodile sont tous deux présents : N' est avalée par $S$ ' comme $\mathrm{N}$ est avalée par Crocodile. $\mathrm{S}$ ' ne s'oppose pas à $\mathrm{S}$ comme le méchant face au bon ; les mythes sont amoraux et ne peuvent prêter à aucun jugement de cette sorte. S' ou Crocodile c'est l'image du «mari» qu'il ne faut pas avoir, ou encore du non-foyer (celui où la femme ne doit pas demeurer). On voit en même temps que l'opposition N-N' demande à être nuancée, comme s'il s'agissait de deux possibilités plutôt que de deux individus.

100 Mais qui est $\mathrm{S}$, ce caractère qui pose un problème (le mythe en pose plus qu'il m'en résout) aux autres personnages du récit et au mythologue bien davantage qu'à l'auditeur averti?

Nous avons vu par quoi tlan peut être remplacé dans d'autres textes du même ensemble mythique. S n'est pas nécessairement un ophidien (d'apparence), il est aussi dragon, homme difforme, anguille, etc. Quand S est un simple mari ordinaire (du genre Homo), en Ms 24, lui correspond, en Mj 115, un S' qui est un seigneur rival. Dans le cycle de Drit, où le héros $\mathrm{N}$ est un jeune homme, le correspondant de $\mathrm{S}$ est une fille-forêt ou une fille-oiseau. En Mj 30, où l'animal se présente d'abord sous une apparence humaine, retournant ainsi le schéma classique, c'est la Dame du Figuier qui correspond à $\mathrm{S}$ dans 
sa fonction de discriminer les deux sœurs. Dans la version «Anguille » (Ms 6, Mj 27) ce personnage unique est lui-même $S$ ou $S$ ' selon l'homme à qui il a affaire. Le rapport Anguille/cadet inverse le rapport Anguille/aîné (ou seigneur), comme (similitude : $\approx$ ) le rapport Dame-Figuier/cadette inverse le rapport Dame-Figuier/aînée, comme S/ cadette inverse S'/aînée :

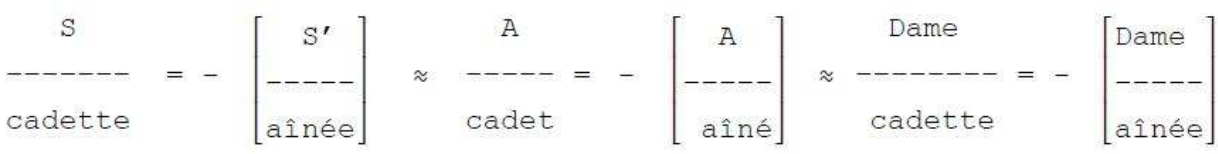

Si c'est bien la relation qui définit les termes, alors $\mathrm{S}$ ne différerait pas plus de S' que A de A.

À côté de ce jeu de remplacements, il nous faut revoir ce que fait un personnage Serpent dans d'autres contextes. Je pense principalement à Mj 50 : ici l'homologue de $\mathrm{S}$ est le propre frère de la jeune fille. Entre deux phases d'existence comme jeune homme il devient serpent d'eau, au fond d'un gouffre, pendant que sa sœur se trouve murée dans une termitière : double impasse d'une situation incestueuse, qui apparente $S$ à $S$ ' (eau) et N à N' (termitière) ; il n'y a plus qu'un couple, totalement ambigu. N'avons-nous pas là, condensée, l'image de l'amour impossible avec un amant trop prochain, le frère, ou trop lointain, la bête? Si nous considérons que les versions du conte-type sont l'extension de cette situation, éclatée en deux couples, S-N et S'-N', alors le mystérieux $S$ s'explique: Homme, mais non pas frère, Bête, en apparence seulement, il serait l'époux possible, à la fois proche et éloigné comme le cousin croisé (celui qui convient le mieux en ces régimes de mariage préférentiel), mais restant ambigu puisque garçon et fille doivent renaître de l'eau (cf. réparation de l'inceste, signalée plus haut), sauf dans les versions böhnar et khmer où l'éclatement est poussé à son comble : N n'a pas à renaître, le couple S-N est parfait, à l'inverse de N' qui disparaît dans les eaux ou devient animal - ce qui rapproche le mariage avec la bête du mariage avec le frère (les incestueux sont traités de « chien et cochon », (Dournes, 1972, 143-144).

Le mariage est un risque, aller au plus près paraît le plus sûr, mais « trop près " c'est comme «trop loin». N et N' sont deux attitudes devant ce risque, et aussi deux attitudes devant l'Autre : être prête à l'aimer ou n'aimer que soi. De toute façon il faut mourir, pour renaître à une nouvelle vie, ou pour être définitivement engloutie. Dans ces conditions, le « paradis » c'est l'Autre, l' « enfer » c'est Soi.

Ainsi se trouverait confirmé ce que je supposais au départ : les paires N-N' et S-S' ne constituent pas quatre individus, mais deux possibilités (essai réussi N-S, essai raté N'$S^{\prime}$ ) de réaliser le couple, entre un jeune homme qui mue et une jeune fille qui n'est (au début) pas amoureuse (parce qu'elle n'a aucune raison de l'être) ; ce n'est que dans la mise en scène du mythe que le jeu se joue à quatre personnages. N-N' et S-S' (paires antithétiques et dédoublées) c'est le moi divisé, qui ne sait pas trop qui il est et ne se réalise qu'en perdant une part de lui-même, s'il en prend le risque. La leçon de l'histoire pourrait être: la vie est faite d'occasions manquées, sauf pour les êtres d'exception - ceux que le mythe illustre justement.

Je n'ai pas tiré parti de tous les éléments que l'analyse a dégagés; tel n'était pas mon propos. Je ne pouvais, dans le cadre de cet article, faire une étude exhaustive d'un thème si grave, lourd de sens (au pluriel) et épaissi par tant d'autres textes que je n'ai pas mentionnés. Je voulais simplement donner un exemple d'une méthode de travail et montrer notamment qu'un corpus de mythes (interethnique mais intra-culturel) forme 
un système; c'est sur ce fond que chaque texte prend une coloration et une signification propres à la population qui le récite, en même temps que l'ensemble acquiert (à un autre niveau) un sens plus profond pour celui qui l'analyse, en connaissance des contextes ethnographiques. C'est une façon de dire que peut être fausse la querelle suscitée autour du "culturalisme »; l'organisation des différences révèle une signification commune. C'est bien pourquoi ces mythes sont traduisibles dans toutes les langues des peuples du monde, chacun l'interprétant à sa façon et toutes ces façons formant à leur tour un système où peuvent se reconnaître des constantes de l'esprit humain.

\section{BIBLIOGRAPHIE}

Austin, J.L., 1970, Quand dire c'est faire, Paris, Seuil.

AYMOniER, E. et CABATON, A., 1906, Dictionnaire čam-français, Publ. EFEO, Paris, Leroux.

Cahiers de littérature orale, $1976, \mathrm{n}^{\circ} 1$.

DetienNe, M., 1977, Dionysos mis à mort, Paris, Gallimard.

DouRnES, J., 1948, Chants antiques de la montagne, Bulletin de la Société des Études Indochinoises, Saigon, XXIV, 3 et 4, p. 1-111.

DouRnES, J., 1953, Le Maître des Eaux (conte montagnard sré), France-Asie, 91, p. 25-29.

DoURNES, J., 1971, Le discret et le continu, JATBA, juillet-août, p. 274-287.

DouRNES, J., 1972, Coordonnées, Structures jörai familiales et sociales, Paris, Institut d'ethnologie.

DOURNES, J. 1975, La fée du figuier, L'Ethnographie, 68, p. 79-92

DOURNES, J., La fée du figuier, L'Ethnographie, 69, p. 81-114.

DOURNES, J., 1976, Le parler des Jörai et le style oral de leur expression, Paris, P.O.F.

DOURNES, J., 1977, Akhan, contes oraux de la forêt indochinoise, Paris, Payot.

GuILLEMINET, P., 1959-63, Dictionnaire bahnar-français, publ. EFEO, Paris, 2 vol.

Guilleminet, P, s.d., Textes Bahnar, Ch. XVI, I, P/16, p. 1000-1025, ms.

KeMLIN, E., 1917, Alliances chez les Röngao, BEFEO, XVII, p. 1-120.

LANDES, A., 1887, Contes Tjames, Saigon.

TOLKIEN, J.R.R., 1949, Farmer Giles of Ham, London, Allen \& Unwin

TOLKIEN, J.R.R., 1964, Tree and leaf, London, Allen \& Unwin. 


\section{NOTES}

1. J'appelle «conte-type " la version qui, en telle ou telle ethnie, se rapproche le plus de ce que j'ai choisi arbitrairement, pour la commodité, comme mythe de référence (sans faire de distinction entre conte et mythe) ; je ne le prends donc pas au sens des folkloristes comparatistes.

2. La séquence (5), présente ailleurs, est absente de cette version.

3. Oublions que le mot est féminin en français ; en fait, il s'agit d'un mâle.

4. Le cham et le khmer, langues à écriture de type indien, sont donnés ici, pour uniformiser la transcription, en phonétique, qui représente la prononciation actuelle; en translittération on écrirait, pour cet exemple, ba's.

5. Nous publions la note de S. Thierry dans la section Discussions, infra, p. 167 (n.d.l.r). [cf. CLO $\left.\mathrm{n}^{\circ} 2,1977\right]$.

\section{RÉSUMÉS}

Cet article propose une analyse très détaillée de quatorze textes provenant de quatre ethnies indochinoises. L'auteur démontre que si l'histoire peut se résumer de la même façon, se réduire à une trame unique, la signification n'est souvent pas la même. Il est important d'en dégager les spécificités culturelles.

This article is a detailled analysis of fourteen texts from four Indochinese ethnic groups. The author demonstrates that while the stories can be summed up the same way, or reduced to a single outline, the meaning is often different. The meaning is found in its cultural specificities.

\section{INDEX}

Keywords : Jörai, Indochina, Variants, Cultural Specificity, Snake, Molt

nomsmotscles Jörai

Index géographique : Indochine

Thèmes : anthropologie (Asie)

Mots-clés : variantes, spécificités culturelles, serpent, mue 